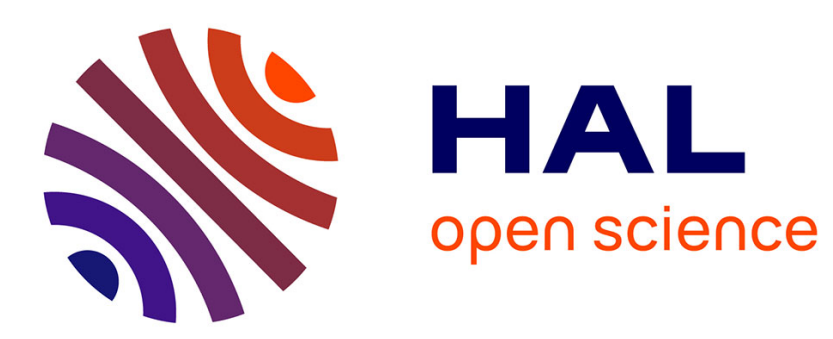

\title{
A model for failure in thermoplastic elastomers based on Eyring kinetics and network connectivity
}

\author{
Stefano Aime, N. D. Eisenmenger, T. A. P. Engels
}

\section{To cite this version:}

Stefano Aime, N. D. Eisenmenger, T. A. P. Engels. A model for failure in thermoplastic elastomers based on Eyring kinetics and network connectivity. Journal of Rheology, 2017, 61 (6), pp.1329-1342. 10.1122/1.5000808 . hal-01653311

\section{HAL Id: hal-01653311 https://hal.science/hal-01653311}

Submitted on 8 Jun 2021

HAL is a multi-disciplinary open access archive for the deposit and dissemination of scientific research documents, whether they are published or not. The documents may come from teaching and research institutions in France or abroad, or from public or private research centers.
L'archive ouverte pluridisciplinaire HAL, est destinée au dépôt et à la diffusion de documents scientifiques de niveau recherche, publiés ou non, émanant des établissements d'enseignement et de recherche français ou étrangers, des laboratoires publics ou privés. 


\title{
A model for failure in thermoplastic elastomers based on Eyring kinetics and network connectivity
}

\author{
S. Aime ${ }^{\text {a) }}$ \\ Laboratoire Charles Coulomb (L2C), UMR 5221 CNRS-Universite de Montpellier, Montpellier 34095, France \\ N. D. Eisenmenger ${ }^{\mathrm{b})}$ and T. A. P. Engels ${ }^{\mathrm{c})}$ \\ DSM Ahead, Material Science Center Urmonderbaan 22, 6167 RD Geleen, the Netherlands
}

(Received 31 March 2017; final revision received 14 August 2017; published 1 November 2017)

\begin{abstract}
A simple model is introduced to describe the failure mechanisms in soft thermoplastic elastomers. In particular, we address the strong embrittlement with increasing temperature observed in strain rate imposed tensile experiments. This behavior is in sharp contrast to classic thermoplastics and seems to be general for these types of systems, irrespective of their exact chemical nature. We show that a kinetic model describing the supramolecular association of hard blocks in terms of an Eyring rate equation captures the correct stress and temperature dependence of failure strain. We model the material as a transient network, whose failure is associated with the loss of connectivity. The network percolation threshold, a key parameter of the model, is studied with numerical simulations, in order to investigate the interplay between structure, connectivity, and mechanical properties. (C) 2017 The Society of Rheology. [http://dx.doi.org/10.1122/1.5000808]
\end{abstract}

\section{INTRODUCTION}

Thermoplastic elastomers (TPEs) are an interesting subset of the engineering plastics family. Their thermoplastic processability in combination with elastomer-like mechanical characteristics $[1,2]$ makes them a valuable addition to the wide range of available polymers. In contrast to the classical thermoset-based elastomers, their thermoplastic nature allows them to be processed just like any other thermoplastic polymer and enables rework and recycle of scrap materials $[3,4]$. While classical thermoset elastomers can be considered as chemically crosslinked polymers operating above their glass transition temperature $\left(T_{\mathrm{g}}\right)$, TPEs should be regarded as physically connected, phase-separated polymers where one phase is in the solid state below its melting temperature $\left(T_{\mathrm{m}}\right)$ and the other phase in its rubbery state above $T_{\mathrm{g}}$ [5-13]. They behave mechanically as elastomers with typically low moduli, high elongations before breaking and an overall rubber-like touch-and-feel. It should be noted though that they typically compete at the higher moduli values with classic elastomers. Most TPEs are block or graft copolymers, which makes it possible to tune the desired properties by altering their chemical composition [2]. In this paper, we will focus on polycondensation-based TPEs composed of poly(butylene terephthalate) (PBT) blocks to make up the solid phase [or hard blocks (HBs)] and polyether-based blocks to make up the soft phase [or soft blocks (SBs)].

\footnotetext{
a) Author to whom correspondence should be addressed; electronic mail: Stefano.Aime@umontpellier.fr

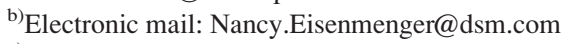

${ }^{c}$ Present address: Eindhoven University of Technology, Postbus 513, 5600 MB EINDHOVEN, Netherlands; electronic mail: Tom.Engels@dsm.com

(C) 2017 by The Society of Rheology, Inc.

J. Rheol. 61(6), 1329-1342 November/December (2017)
}

The deformation of these systems has been quite extensively studied in literature, especially in connection to their morphology [9,13-20]. Most of these studies, however, focused on the behavior at room temperature, and did not describe the effect of temperature on the mechanical properties of the materials, which we address. Quite generally stated, we observe that soft TPEs, characterized by a low content of HBs, below 50\%, show an unexpected, and often undesired, drop of extensibility as temperature is increased. This happens in the absence of any thermodynamic or structural transition, and it is in sharp contrast with standard thermoplastic materials, which are rather fragile at low temperatures and increase their ductility when heated as a consequence of the enhanced chain mobility [21,22]. Despite its relevance for applications, to the best of our knowledge this observation has not been studied explicitly in the literature, although some papers do show similar observations [20,23-25]. We find this behavior to be general for this type of systems, irrespective of their exact chemical nature (polyester, polyurethane, and polyamide based systems all show the same phenomenology), and from a fundamental point of view this represents an important challenge for theoretical models describing failure in TPEs.

The rich morphology displayed by these systems is discussed in detail in a paper by Gabriëlse et al. [26] where a PBT-poly(tetramethylene oxide) (PTMO) system is investigated. The block-copolymer nature suggests the material to consist of two phases, but it is shown that depending on the chemical affinity of the two components the phase behavior can be more complex. In the case discussed in that paper, up to five distinct phases are identified: (1) a pure PBT crystalline phase, (2) a glassy PBT phase, (3) a mixed PBT-PTMO glassy phase, (4) a rubbery PTMO phase, and (5) a crystalline PTMO 
phase. Here we will not go into that level of detail and for the sake of simplicity we will treat these systems as simple binary systems, made by crystalline PBT and rubbery SB material.

In this paper we present a kinetic model, qualitatively accounting for the embrittlement of TPEs at high temperatures. The paper is structured as follows: after a first overview of available experimental observations (Sec. III), the kinetic model is presented and qualitatively validated (Sec. IV). For a more detailed comparison with experimental data, in Sec. V, we present an implementation of this kinetic model on a cubic lattice. A conclusive section closes the paper, with a general discussion of the main results.

\section{MATERIALS AND METHODS}

We use as model systems two different, specifically synthesized poly(ether-ester) block-copolymers based on PBT as HB, and PTMO or poly(ethylene glycol) (PEG) as SBs. The details of the two systems are reported in Table I. The PEG-based system is used to study the effect of molecular weight, and to that aim the synthesis is stopped at a relatively low molecular weight and subsequently grown in discrete steps of molecular weight by solid state postcondensation at high temperature under dry nitrogen conditions [27]. Molecular weights are determined using size-exclusion chromatography in hexafluoroisopropanol (HFIP).

Materials are compression molded into $200 \mu \mathrm{m}$ thick sheets at a temperature of $225^{\circ} \mathrm{C}$. Materials are allowed to equilibrate shortly in the order of minutes at this temperature before being cooled down to room temperature by cooling the press by an internal water circulation system. Tensile bars of ISO 527/1BA standard shape are then punched from those sheets and tested on a standard Zwick Z010 Universal tensile testing machine. In all tests, the samples are equilibrated at target temperature for $10 \mathrm{~min}$. For a tensile test, they are subsequently stretched at a constant engineering strain rate till failure. In the case of a creep experiment, the load is applied within $10 \mathrm{~s}$ after which it is held constant until final failure. Strain rates and temperatures are indicated where appropriate in the respective figures and/or captions. Stresses and strains are reported as engineering values unless stated otherwise.

The differential scanning calorimetry (DSC) experiments are performed on a Mettler-Toledo DSC823e equipped with a FRS5 sensor. Calibration was performed by melting peaks of tin and zinc. Scans were performed at heating rates of $20 \mathrm{~K} / \mathrm{min}$. Standard $40 \mu \mathrm{l}$ aluminum crucibles are used and samples weighed approximately $5-10 \mathrm{mg}$. Nitrogen is used as a purge gas.

TABLE I. Specifications of the two model systems studied in this paper.

\begin{tabular}{lccc}
\hline \hline & PTMO-based & PEG-based & \\
\hline & $30 / 70$ & $50 / 50$ & HB/SB mass ratio \\
$M_{\mathrm{HB}}$ & $880 \mathrm{~g} / \mathrm{mol}$ & $1000 \mathrm{~g} / \mathrm{mol}$ & HB molecular weight \\
$M_{\mathrm{SB}}$ & $2000 \mathrm{~g} / \mathrm{mol}$ & $1000 \mathrm{~g} / \mathrm{mol}$ & SB molecular weight \\
$M_{w}$ & $60 \mathrm{~kg} / \mathrm{mol}$ & Various & Total molecular weight (weight average) \\
$T_{g}$ & $-80^{\circ} \mathrm{C}$ & $-50^{\circ} \mathrm{C}$ & Glass transition temperature \\
$T_{m}$ & $170^{\circ} \mathrm{C}$ & $194^{\circ} \mathrm{C}$ & Melting temperature \\
\hline \hline
\end{tabular}

\section{EXPERIMENTAL OBSERVATIONS}

Figure 1 shows representative engineering stress-strain curves obtained for the PTMO-based system at different temperatures. After a first linear regime, lasting only a few percent deformation (hardly distinguishable in the figure), we observe a broad yield range, around $10 \%$ deformation, where plastic deformation causes the slope of stress-strain curves to decrease. This is followed by an ongoing deformation at mildly increasing stress levels until strain hardening is eventually observed at higher deformations, more pronounced at lower temperatures. In this case, strain hardening is enhanced by strain-induced crystallization of SBs [18] (indeed, the melting point for strained PTMO chains is around $30^{\circ} \mathrm{C}$ ). Finally, engineering stress drops as fracture is abruptly encountered. The fracture event occurs earlier at higher temperatures: for example, a sample can withstand a strain higher than $1000 \%$ at $50^{\circ} \mathrm{C}$, whereas failure is observed at only $300 \%$ deformation at $125^{\circ} \mathrm{C}$. Remarkably, as one can see from DSC [Fig. 1(b)], the melting temperature of the system is $170^{\circ} \mathrm{C}$, and no additional thermodynamic transitions are observed in the temperature range where tensile experiments are performed. The possibility of straininduced crystallization being connected to the observed reduction in ductility with increasing temperature was ruled out using observations not reported in this paper. Indeed, repeating the same experiments on other TPEs based on softblocks that do not exhibit strain-induced crystallization, we could observe a very similar behavior. Therefore, this embrittlement at high temperature, in the absence of any thermodynamic signature, appears to be general for soft TPEs, and it represents the first observation that we want to model.

The influence of strain rate on the stress-strain curves was also tested. Figure 2 shows representative curves obtained by

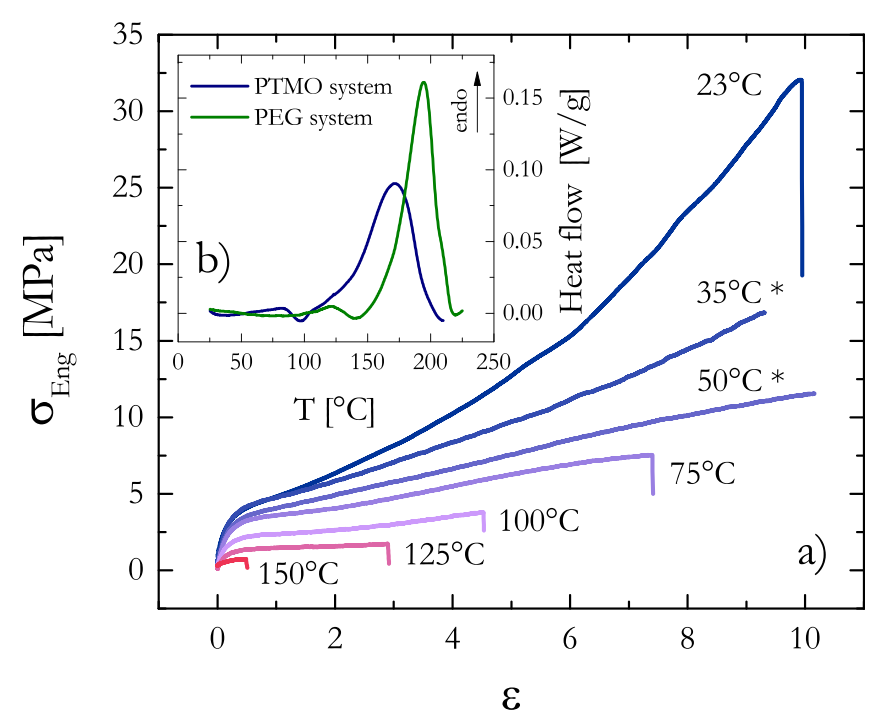

FIG. 1. (a) Engineering stress-strain curves of the PTMO-based system at different temperatures, as indicated in the labels $(T=23,35,50,75,100,125$, and $150^{\circ} \mathrm{C}$ from blue to red), for a fixed strain rate $\dot{\varepsilon}=0.17 \mathrm{~s}^{-1}$. Experiments at 35 and $50{ }^{\circ} \mathrm{C}$ never reached failure, because of the limited strain range of the tensile machine used. (b) DSC traces measured on heating at $20 \mathrm{~K} / \mathrm{min}$. An arbitrary baseline was subtracted from the experimental data. 


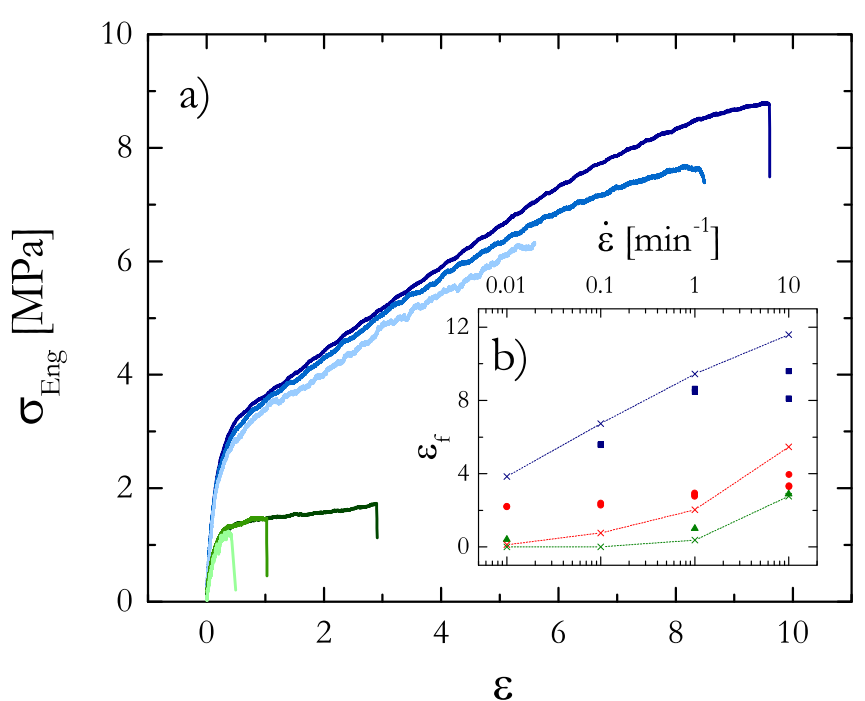

FIG. 2. (a) Representative engineering stress-strain curves obtained experimentally for PTMO-based system at various temperatures (blue: $60^{\circ} \mathrm{C}$, green: $125^{\circ} \mathrm{C}$ ) and strain rates (decreasing from dark to light). (b) Symbols: maximum elongation at break as a function of the strain rate for the same temperatures (red data: $T=100^{\circ} \mathrm{C}$, not shown in the main graph). Crosses and dashed lines: elongation at break obtained by simulations using the experimental stress-strain curves as input. Chain length is $N=9 \mathrm{SBs}$, and kinetic parameters are $\Delta H=105 \mathrm{~kJ} / \mathrm{mol}$ and $v^{*}=2.2 \times 10^{-4} \mathrm{~m}^{3} / \mathrm{mol}$.

repeating the experiment at one temperature for different values of the imposed strain rate. The experimentally applied strain rates ranged from a maximum of $10 \mathrm{~min}^{-1}$ to a minimum of about $0.001 \mathrm{~min}^{-1}$ (i.e., from 0.17 to $1.7 \times 10^{-5}$ $\mathrm{s}^{-1}$ ). Experimental data show that at temperatures above $50{ }^{\circ} \mathrm{C}$, the stress is roughly independent of strain rate, suggesting that the characteristic timescales relevant for stress relaxation should fall outside the range of accessible timescales $\left(\dot{\varepsilon}^{-1}\right)$. Nevertheless, one can see from Fig. 2(b) that the elongation at break shows a weak but clear dependence on $\dot{\varepsilon}$, which is the second observation we would like to capture.

Finally, we address the impact of chain length on mechanical properties. For this reason, PEG-based systems of different molar masses are tested, and the tensile curves obtained are shown in Fig. 3. While the linear regime seems to be roughly the same for all samples, strong differences are observed with respect to extensibility and stress levels: as $M_{w}$ is increased, the failure strain $\varepsilon_{f}$ sharply increases until a plateau is reached. At room temperature, samples with higher molar mass also show a strong strain hardening profile, so that toughness is an increasing function of $M_{w}$.

To summarize, the experimental observations that we would like to understand and capture with a phenomenological model are the following:

(1) Elongation at break drops dramatically with increasing temperature (Fig. 1).

(2) Elongation at break also decreases with decreasing strain rate, and the dependence is weaker at lower temperatures (Fig. 2).

(3) Elongation at break increases with increasing chain length and then it saturates to a $M_{w}$-independent value (Fig. 3).

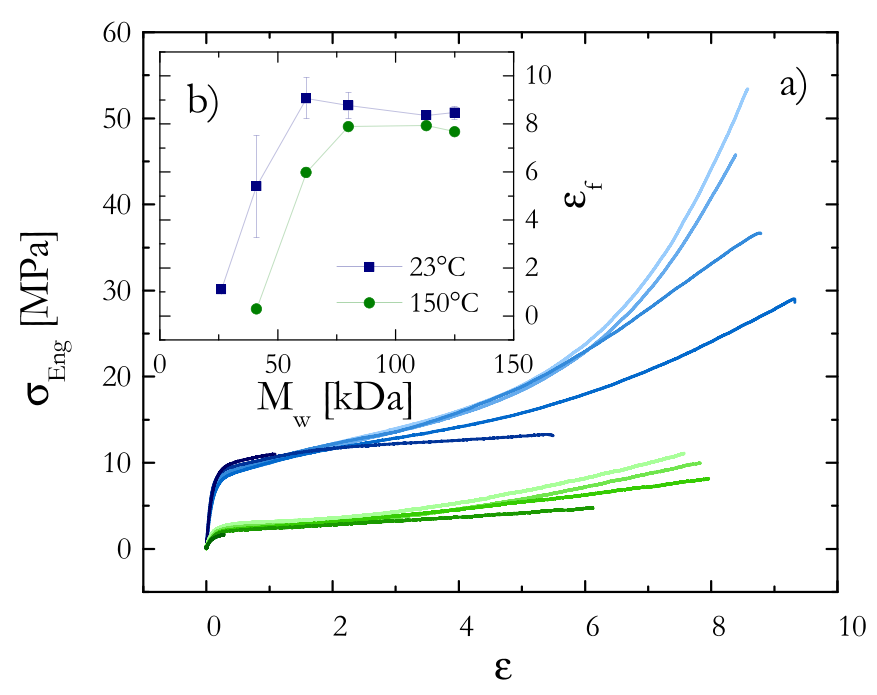

FIG. 3. (a) Engineering stress-strain curves obtained with the PEG-based system, with increasing molar mass (weight average molecular weights $M_{w}=26,41,62,80,113$, and $125 \mathrm{kDa}$ ) from dark to light, at two representative temperatures $23^{\circ} \mathrm{C}$ (blue) and $150^{\circ} \mathrm{C}$ (green). Strain rate is $\dot{\varepsilon}=0.17 \mathrm{~s}^{-1}$. (b) Elongation at break as a function of $M_{w}$. Error bars are included to indicate the variability on the data.

\section{KINETIC MODEL}

\section{A. Sample structure}

As briefly discussed in the Introduction, at room temperature and in the absence of mechanical constraints, TPEs have a complex morphology: SBs form an amorphous matrix surrounding crystalline domains composed of HBs, which are usually entangled or connected, and form the main stressbearing structure in the linear regime $[11,19]$. This picture in both PTMO and PEG systems holds between $T_{g} \approx-60^{\circ} \mathrm{C}$ and $T_{m} \approx 170-200^{\circ} \mathrm{C}$ (cf. Table I), which is the working temperature range of these materials.

Under stress the morphology changes differently for soft grades (mainly composed of SBs) and hard grades (mainly composed of HBs). Direct experimental observations $[9,15,17-20,28-31]$ show that soft TPE materials break their network of HB crystals and develop a characteristic fibrillar structure when stretched beyond 100\%-200\% strain. In such a structure, to a lesser extent found in harder TPEs, crystalline domains formed by HBs tend to align in planes perpendicular to the strain direction, whereas SBs, bridging different crystalline domains, are progressively stretched.

The fact that the observed drop of $\varepsilon_{f}$ is only observed on soft TPE grades suggests that the failure mechanism might be linked to this fibrillar structure, that we take as a starting point for the description of failure mechanisms.

\section{B. Failure mechanism}

In analogy to recent studies on transient networks [32-34], our model describes failure in terms of connectivity loss: the sample will break as soon as two parts get disconnected. Connectivity is thus a key element in our model, and it will be evaluated within the framework of percolation theory. For the sake of simplicity, we assume that the main features of material failure do not rely on the effect of eventual entanglements between SBs, which are neglected here. 
Therefore, we attribute the sample's initial connectivity to physical bonds that can be formed between HBs: as long as this network of bonds persists, the structure will be able to withstand the applied load. However, physical bonds are dynamic: they can break and reform as a consequence of temperature and stress. At rest, a dynamic equilibrium is reached, with breaking events balancing the bond formation, but an external stress biases this equilibrium, favoring breaking processes over the reformation: this causes HB crystal fragmentation and weakens the network by reducing the amount of elastically active, stress-bearing chains [32]. In analogy with established work in literature, mainly focusing on yielding [35-42] or creep failure [42-49], we assume that the kinetics for inter-HB bond breaking and reformation can be modeled using Eyring's rate equation [50]:

$$
k(\sigma, T)=k_{0} \exp \left(-\frac{\Delta G}{k_{B} T}\right) \sinh \left(\frac{\sigma v^{*}}{k_{B} T}\right),
$$

where $k_{0}$ represents a reference rate, $k_{B}$ is Boltzmann constant, $\Delta G$ is the activation energy of bond breaking, and $v^{*}$ is the activation volume.

The Eyring rate equation was originally developed to describe chemical reaction rates, but it was soon extended to describe physical processes like viscous flow [51-53]. Since then, it has been widely applied to polymer yielding and plastic flow, and most of the time the rate constants involved in the model were used to describe the rate of flow [42,54-56]. Instead, here we use Eyring rate constants to model the breakdown of those bonds capable of supporting an applied load. In this regard, our application of Eyring theory is less conventional, and closer to the one proposed by Matz et al. [49] or Zhurkov [57,58], who present a mathematical framework close to that of Eyring, although it should be noted that in the latter case the author focuses on chain scission as the dominant cause for failure. On the other hand, in the present study the absolute rate $k(\sigma, T)$ describes the evolution of the amount of associated physical bonds (rather than chemical bond breaking). Its value at rest $k_{T}=k(\sigma=0$, $T$ ) and below $T_{m}$ is typically such that almost all HBs are associated at equilibrium [11]. However, the bias introduced by stress shifts the fraction $A$ of associated HBs toward lower values, until the system eventually becomes disconnected: as strain increases, we can describe plasticity in terms of a function $A(\varepsilon)$ decreasing from 1 to a critical value $A_{t h}$, which we will treat as a percolation threshold. Therefore, within this framework, elongation at break can be computed as the strain $\varepsilon_{f}$ at which $A\left(\varepsilon_{f}\right)=A_{t h}$.

The evolution of $A(\varepsilon)$ can be computed using Eq. (1), by assuming a first order process for bond breaking and integrating the differential equation

$$
\frac{d A[\varepsilon(t)]}{d t}=-k[\sigma(t)] A[\varepsilon(t)] .
$$

In order to obtain an analytical solution, an expression for the stress history $\sigma(t)$ is required. This can be easily done in creep [21,43], whereas the calculation is less straightforward when a constant strain rate is imposed. The simplest assumption, which we will use here, is to require that a simple affine relation between true stress $\sigma_{\text {True }}$ and strain holds beyond the yield point of the material: $\sigma_{\text {True }}(t)=\sigma_{0}+\sigma_{1} \varepsilon(t)$, where $\varepsilon(t)=\dot{\varepsilon} t$, and $\sigma_{0}$ and $\sigma_{1}$ are phenomenological parameters extracted from fits to the individual tensile curves [Fig. 4(a)]. It is clear from this graph that the chosen relation for stress as a function of strain is less than optimal, and, e.g., a fit using a neoHookean scaling will result a strongly improved fit [Fig. 4(b)]. However, to allow for an analytical solution to exist we have to compromise on this accuracy and suffice with a simple, less accurate description. Note that toward higher temperatures this assumption is more accurate compared to lower temperatures.

Moreover, we also assume that because of internal stresses two HBs that detach from each other are pulled apart, and will not recombine: under this assumption the hyperbolic sine in Eq. (1) is simplified to a single exponential. This second assumption is not necessary in itself, but makes the calculations easier.

By solving the differential equation with initial condition $A(0)=1$, we obtain

$$
A(\varepsilon, \dot{\varepsilon})=\exp \left[-\frac{k_{0}}{\dot{\varepsilon}} \frac{k_{B} T}{v^{*} \sigma_{1}} e^{-\left(\Delta G-v^{*} \sigma_{0}\right) /\left(k_{B} T\right)}\left(e^{\left(v^{*} \sigma_{1} / k_{B} T\right) \varepsilon}-1\right)\right] .
$$

Thus, once an assumption on $A_{t h}$ is made, the elongation at break $\varepsilon_{f}$ can be easily calculated by inverting this expression

$$
\varepsilon_{f}=\frac{k_{B} T}{\sigma_{1} v^{*}} \ln \left[1-\frac{\dot{\varepsilon}}{k_{0}} \frac{v^{*} \sigma_{1}}{k_{B} T} e^{\left(\Delta G-v^{*} \sigma_{0}\right) /\left(k_{B} T\right)} \ln A_{t h}\right] .
$$

\section{Model validation}

Figure 5 shows a set of $\varepsilon_{f}$ values obtained for one choice of model parameters and plotted as a function of temperature for different strain rates. A qualitative comparison with experimental data shows that

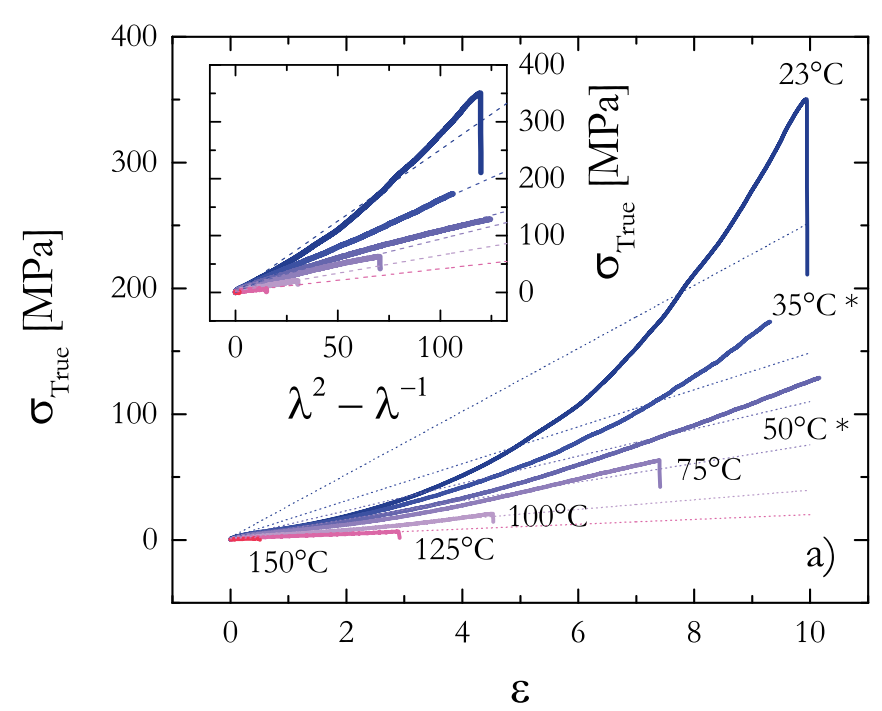

FIG. 4. (a) Experimental data of Fig. 1 (solid lines) along with linear fits (dashed lines) of true stress versus strain. (b) Alternative scaling according to neo-Hookean relation. 


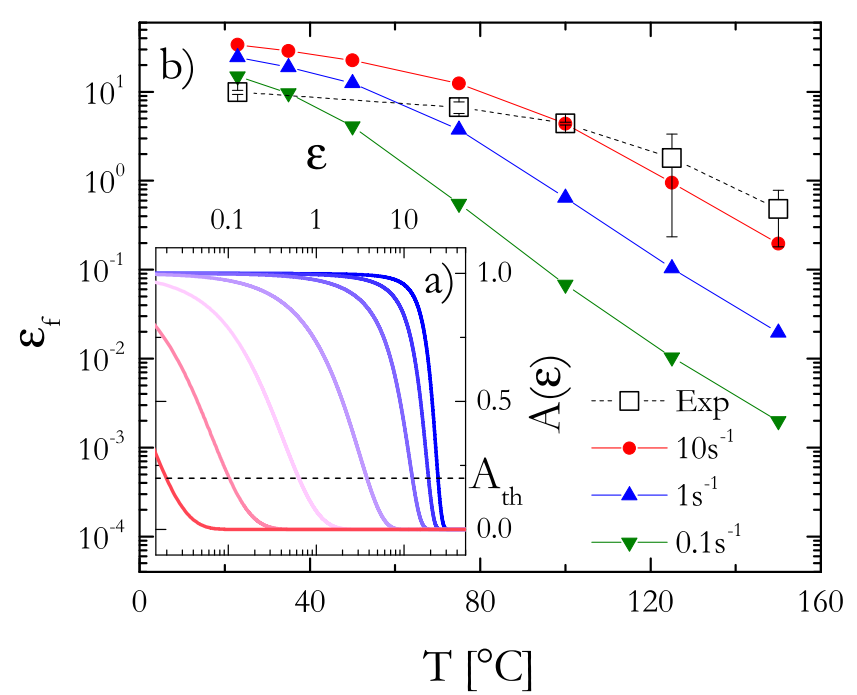

FIG. 5. (a) Predicted evolution of associated fraction $A(\varepsilon)$ as a function of the engineering strain. Model parameters used are $\Delta G=105 \mathrm{~kJ} / \mathrm{mol}$, $v^{*}=2.210^{-4} \mathrm{~m}^{3} / \mathrm{mol}, \sigma_{0}=\sigma_{1}=3 \mathrm{MPa}, \dot{\varepsilon}=1 \mathrm{~s}^{-1} T$ is increasing from blue to red. (b) Predicted strain at break $\varepsilon_{f}$ obtained for a choice of $A_{t h}=0.2$, plotted as a function of temperature and for different strain rates, compared to experimental values extracted from Fig. 1. Error bars represent data dispersion evaluated on 2-6 independent measurements (data not shown).

(1) By fixing $A_{t h}$ and varying $T$, it is possible to reproduce the drop in $\varepsilon_{f}$ with increasing temperature. For the highest temperatures, we get $\varepsilon_{f} \propto T^{-1}$, whereas at lower temperatures, the $T$ dependence is weaker.

(2) The trend with decreasing $\dot{\varepsilon}$ also resembles the one observed experimentally: Eq. (4) predicts $\varepsilon_{f} \propto \dot{\varepsilon}$ for high temperatures, whereas $\varepsilon_{f} \propto \ln (\dot{\varepsilon})$ at low temperatures. In any case, the effect of increasing temperature on reduction in elongation at break is stronger than the effect of increasing strain rate.

(3) Since longer chains (with higher number of HBs per chain) form a better connected structure, the effect of increasing molecular weight may be schematized as a decrease in $A_{t h}$, which in turn increases $\varepsilon_{f}$ (data not shown), as qualitatively observed in Fig. 3.

These observations suggest that our model is able to capture the main features of the observed failure. A next, natural step would be to quantitatively compare our experimental data with predictions based on Eq. (4): this requires an estimation of our model parameters. While $\sigma_{0}$ and $\sigma_{1}$ can be extracted from a linear fit of the tensile curves, the determination of the remaining three parameters $\left(\Delta G, v^{*}\right.$ and $\left.A_{t h}\right)$ is more delicate. In particular, the physical interpretation of $A_{t h}$ may be questionable: in fact, as the deformation is localized around sample defects (in particular during the latest stages before failure), the macroscopic stress measured experimentally deviates from the local stress actually experienced by polymer chains, so that in principle a direct link between the macroscopic stress and the local amount of broken bonds does not exist, and $A_{t h}$ appears to be rather ill-defined. For this reason, in a first instance we assume that the sample deformation is homogeneous, and that bond breaking events occur randomly and homogeneously inside the sample.
Numerical simulations shown in Sec. V will help us quantifying to what extent this assumption is acceptable.

Under this approximation, $\Delta G, v^{*}$ and $A_{t h}$ can be extracted by a using the fits of tensile curves at different strain rates and temperatures as input. In its simplest version, the fitting procedure works as follows: once a set $\left\{\sigma\left(\varepsilon, T_{i}, \dot{\varepsilon}_{i}\right)\right\}$ of tensile curves is provided, we extract from it the mechanical parameters $\sigma_{0}\left(T_{i}, \dot{\varepsilon}_{i}\right), \sigma_{1}\left(T_{i}, \dot{\varepsilon}_{i}\right), \varepsilon_{f}^{(o b s)}\left(T_{i}, \dot{\varepsilon}_{i}\right)$. Then, a reasonable set of kinetic parameters $\left(\Delta G^{(0)}, v^{*(0)}\right.$, $\left.A_{t h}^{(0)}\right)$ is taken as a starting input, and used to compute, for each $\left(T_{i}, \dot{\varepsilon}_{i}\right)$, an expected strain at break $\varepsilon_{f}^{(0)}\left(T_{i}, \dot{\varepsilon}_{i}\right)$. A Monte Carlo algorithm is then used to find the $\left(\Delta G, v^{*}, A_{t h}\right)$ set that provide the best set $\left\{\varepsilon_{f}^{(f i t)}\left(T_{i}, \dot{\varepsilon}_{i}\right)\right\}$ of predicted elongations at break, i.e., the choice that minimizes the $\chi^{2}$ parameter defined as $\chi^{2}=\sum_{i}\left\{\left[\varepsilon_{f}^{(o b s)}\left(T_{i}, \dot{\varepsilon}_{i}\right)-\varepsilon_{f}^{(f i t)}\left(T_{i}, \dot{\varepsilon}_{i}\right)\right]^{2} / \varepsilon_{f}^{(f i t)}\left(T_{i}, \dot{\varepsilon}_{i}\right)\right\}$.

As an example, the fitting parameters extracted for the PTMO-based systems are $\Delta G=105 \mathrm{~kJ} / \mathrm{mol}, v^{*}=2.2 \times 10^{-4}$ $\mathrm{m}^{3} / \mathrm{mol}$, and $A_{t h}=0.2$. We included the experimental strainsto-failure versus temperature of Fig. 1 in Fig. 5 to enable a direct comparison to experimental data. We show that, as stated before, with the chosen parameters, we can describe the trend of decreasing strain-at-break with temperature well and that the absolute values are captured to a reasonable approximation. Toward the lower temperatures, we see the results to differ more; this might be related to the poorer fit to experimental stress-strain curves as shown in Fig. 4(a).

We find that $\Delta G$ is temperature independent, which is an indication that the entropy variation should be negligible with respect to enthalpy. Although it is difficult to quantitatively relate those parameters to some a priori knowledge about the system [59] (we will limit ourselves to treat them as fitting parameters), it is worth mentioning that the activation energy $\Delta G$ and the activation volume $v^{*}$ are comparable in magnitude to what is found in literature for other deformation mechanisms $[39,43,47,49,56,60]$, while the percolation threshold $A_{t h}$ is close to the one extracted by numerical simulations (see Sec. V).

\section{Numerical integration}

Once $\Delta G$ and $v^{*}$ are known, we can refine our analysis by dropping the (strong) assumption about the stress-strain affine relationship: starting from the experimental $\sigma(t)$, Eq. (2) can be numerically integrated, and the full $A(\varepsilon)$ profile can be extracted from each tensile curve. Such a result, for the experimental stress-strain curves of Fig. 1, is represented in Fig. 6(a).

Looking at that figure, one can notice that, for temperatures high enough, sample failure occurs as soon as the fraction of broken bonds approaches a threshold value $1-A_{\text {th }}$ close to $100 \%$, and only weakly dependent on strain rate and temperature. We interpret the fact that low temperatures represent an exception, and that failure apparently occurs later than expected, as a direct consequence of strain hardening: in this case, strain-induced crystallization of SBs may represent an additional contribution to connectivity, not considered in our model. 
$\varepsilon$

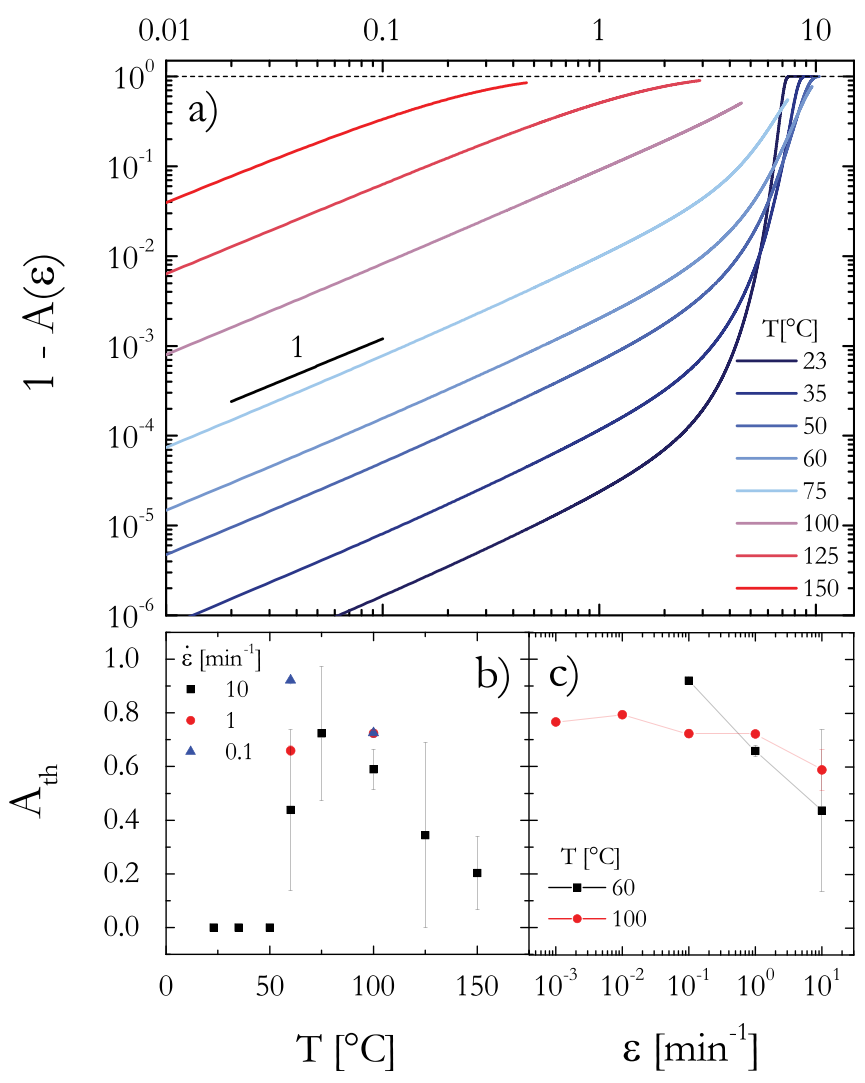

FIG. 6. (a) Fraction of broken bonds as a function of engineering strain, obtained by numerically integrating the differential equation Eq. (2) with $\sigma^{\circ}(t)$ from the tensile curves of Fig. 1. (b) and (c) Percolation threshold $A_{t h}$ (extracted by measuring $A$ at break) plotted as a function of temperature and strain rate.

A more detailed analysis of Fig. 6(a) reveals the existence of two different regimes: for high temperatures $\left(T \geq 100^{\circ} \mathrm{C}\right)$ the fraction of broken bonds grows linearly with time (or strain) throughout the whole experiment. In this temperature range, $\sigma v^{*}$ is always smaller than $\Delta G$, and the bond breaking events are essentially thermally activated. This corresponds to the region where $\varepsilon_{f} \propto \dot{\varepsilon} T^{-1}$ in Eq. (4). On the other hand, at lower temperatures, this regime is eventually interrupted by a second regime, where bond breaking becomes mainly stressactivated and accelerated with respect to thermal activity. This typically happens at a few strain units deformation, when $v^{*}$ eventually becomes larger than $\Delta G$ : as a consequence, in this regime $\varepsilon_{f}$ increases only weakly with decreasing $T$ or increasing $\dot{\varepsilon}$. Thus, the numerical approach not only allows to take into account the detailed shape of the stress-strain curve, but it also provides a valuable insight into plastic activity occurring during deformation.

Furthermore, the numerical approach has a third major advantage: it does not require any assumption on the exact $A_{t h}$ value. Indeed, $A_{t h}$ is no longer a model parameter, but can be extracted by looking at the minimum value reached by $A(\varepsilon)$ before failure. Figures 6(b) and 6(c) show $A_{\text {th }}$ as a function of temperature and strain rate. As one can see, for temperatures higher than $60^{\circ} \mathrm{C} A_{t h}$ is roughly strain rate independent but displays a clear decreasing trend with increasing temperature. This trend cannot be described by
Eq. (4), that assumes a unique $A_{t h}$ value for all temperatures and strain rates. That is why Sec. V will be dedicated to numerical simulations, which will help to attribute such a temperature dependence of $A_{t h}$ to the heterogeneity of sample deformation.

\section{SIMULATIONS}

Until now, homogeneity of sample deformation and plastic activity has been assumed. In this section, we want to discuss the limits of these assumptions, with the help of numerical simulations.

\section{A. General concept}

Our numerical simulations are inspired by, and loosely based on, the work of Mora [33], and might show some resemblance to the work of Termonia [61], although the underlying physics are different. The kinetic model is implemented on a 3D cubic lattice, as shown in Fig. 7(a): each site $(i, j, k)$ represents a HB that can be bound either chemically (via a SB) or physically (via weaker supramolecular interactions) to neighbor HBs, with periodic boundary conditions (PBC) along $\hat{\imath}$ and $\hat{j}$ (strain is applied in the $\hat{k}$ direction). Physical bonding is only allowed within $(i, j)$ planes, that represent $\mathrm{HB}$ crystals in the stretched fibrillar structure, whereas chemical bonds can develop both in the strain direction (in this case they will be elastically active, bridging two different crystals) and in the $(i, j)$ plane (in this case they will behave as loops, not contributing to the sample modulus). Thus, within this model, a block copolymer containing $N$ SBs and $N+1 \mathrm{HBs}$ is a $N$-step self-avoiding walk on the lattice. Chemical bonds are permanent, whereas physical bonds can randomly break and reform following the rate given by Eq. (1). Sample connectivity is evaluated in two steps [Figs. 7(b) and 7(c)]: in a first step single crystals are identified in each $(i, j)$ plane. In this model, a crystal is a set of sites connected with each other but disconnected from all the others belonging to the same $(i, j)$ plane. Along the transversal $(i, j)$ directions, PBC are taken. In the second step, the connectivity of crystals belonging to consecutive $(i, j)$ planes is checked: two crystals will be connected if and only if there is at least one chemical bond (i.e., a SB) binding one site of the first crystal with another site of the second. If a percolating set of crystals exists bridging the two opposite surfaces of the sample, then the system will be considered as connected.

The boundary conditions for the model are prescribed by applying a stress that is either constant in the case of creep simulations (Sec. V B), or increasing with time, following the experimental tensile curves (as described in Sec. V D), in the case of tensile tests. In both cases, starting from a fully connected system and breaking supramolecular bonds, connectivity is progressively lost: disconnected or dangling regions are created [Fig. 7(c)] at the expense of the stressbearing backbone. As a consequence of local mechanical equilibrium, stress is concentrated in the regions with the highest density of disconnected sites, which in turn increases the bond breaking rate in that same region: the fluctuations in 


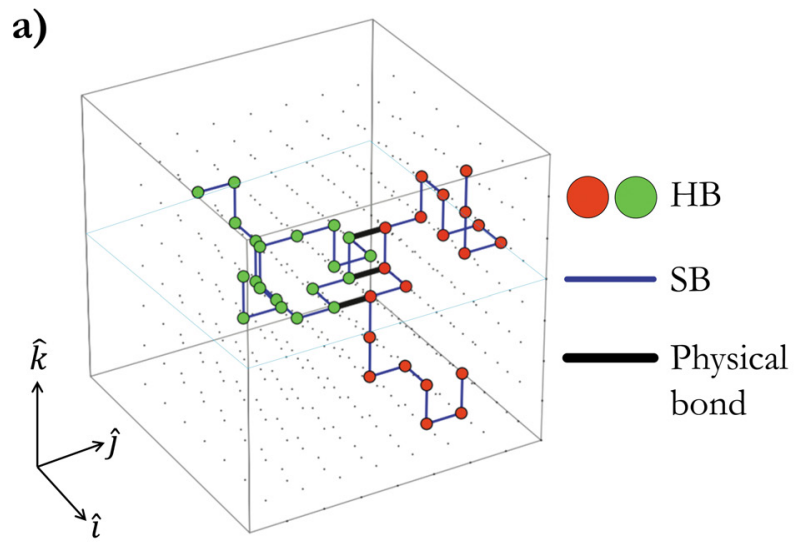

b)

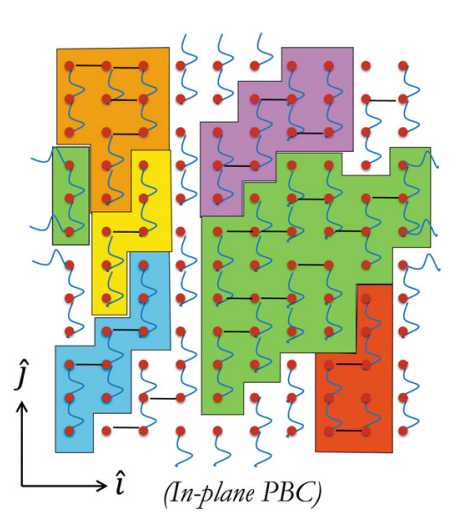

c) Longitudinal:

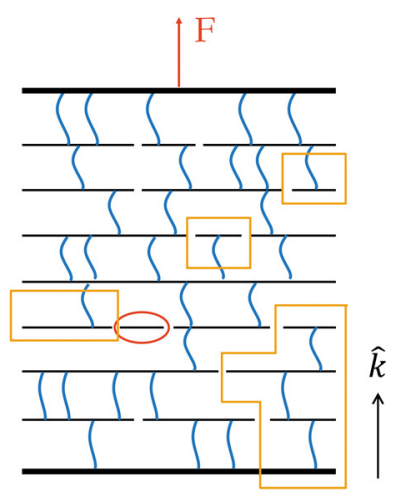

FIG. 7. (a) Sketch of the simulation box filled with two copolymer chains. The stress is applied along the vertical direction. HB segments occupy lattice sites. Segments belonging to different chains are shown in different colors for the sake of clarity. SB segments are represented as blue segments, and can assume all orientations, whereas physical bonds (black segments) only develop in horizontal planes, perpendicular to the stretching direction, in order to mimic the fibrillar structure with oriented HB crystals bridged by SBs. In simulations all lattice sites are occupied by HB segments. (b) Evaluation of transversal connectivity: each horizontal plane is divided in disconnected domains, representing single crystalline fragments. Some of those are highlighted with different colors. PBC are taken in the $(i, j)$ plane. (c) Longitudinal projection of the sample. Horizontal planes are divided in multiple domains that can eventually be connected to adjacent domains along the vertical direction by SB segments. Disconnected domains (highlighted in red) and dangling (orange) ones do not contribute to the stress bearing structure, and are neglected.

the local number of elastically active chains bring information about stress localization and deformation heterogeneity.

\section{B. Tensile creep}

Since our kinetic model is governed by Eyring's rate equation [Eq. (1)], which contains stress but no (explicit) strain dependence, creep appears to be the most natural protocol to test the validity of the model. To support that creep failure of TPEs is also governed by an activated process, a limited set of creep experiments were performed on the PTMO system at elevated temperatures, that yield a creep failure time decreasing exponentially with the applied stress, as one would expect from Eq. (1), and decreasing as well with increasing temperature (data not shown), which is also in line with expectations $[21,43,44,46,48,49,55,62]$. A representative set of creep curves is shown in Fig. 8.

Although numerical simulations cannot grasp the qualitative shape of the full creep curve (they do not contain details about linear viscoelasticity and morphology evolution), they can describe how the failure time varies with stress and temperature (Fig. 9).

For a given applied stress at a fixed temperature, repeated simulations on equivalent samples produce a Weibull distribution of failure times $P\left(t_{f}\right)$ [Fig. 9(a)] [63-66], characterized by an average value $\tau_{f}$ and a shape parameter $\beta$,

$$
P\left(t_{f}\right)=1-e^{-\left(t_{f} / \tau_{f}\right)^{\beta}}
$$

The average failure time $\tau_{f}$ exhibits a sharp dependence on both temperature and applied stress, reflecting the activated nature of failure process $[67,68]$ : both effects can be taken into account by considering an adimensional stress $\tilde{\sigma}=\sigma / \sigma_{T}$, where $\sigma_{T}=k_{B} T=v^{*}$ is a temperature dependent reference stress for stress-activated dynamics. Indeed, once the Arrhenius temperature dependence is taken into account by rescaling time with the thermal rate $k_{T}=k_{0} \exp \left(-\Delta G / k_{B} T\right)$, $\tilde{\sigma}$ allows a collapse of all $\tilde{\tau}_{f}=k_{T} \tau_{f}(\sigma, T)$ data onto a mastercurve, close to the theoretical expectation $\tilde{\tau}_{f}=\tilde{\tau}_{0} / \operatorname{sinc}(\tilde{\sigma})$ [Fig. 9(d)] [43]. The difference between the master curve obtained by simulations and the theoretical function calculated from Eq. (1) is presumably related to the heterogeneity of the deformation observed in simulations. As a consequence of stress concentration, in fact, bond breaking events tend to be localized around the weakest regions, and this causes sample failure to occur earlier than predicted. This effect, more pronounced for large stresses and low temperatures (since in the opposite regime thermal processes push in the direction of a more homogeneous deformation), can be

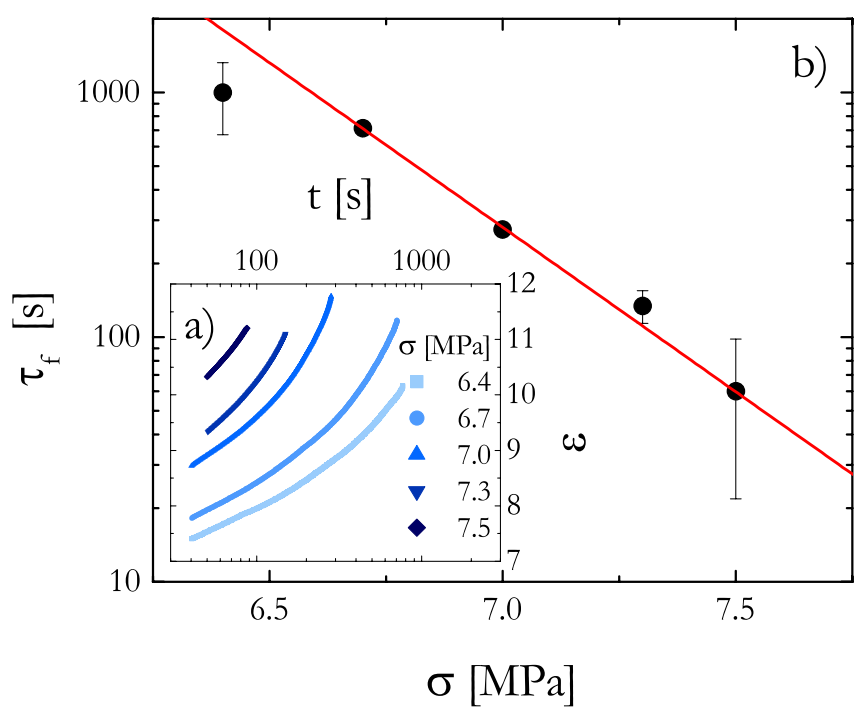

FIG. 8. (a) Representative creep deformations for the PTMO-based system as a function of time since step stress application for different stress values, as indicated in the legend, at a temperature $T=60^{\circ} \mathrm{C}$. (b) Symbols: failure times average on three repetitions of the same experiment, as a function of the applied stress. Error bars represent data dispersion. Red line: exponential fit. 


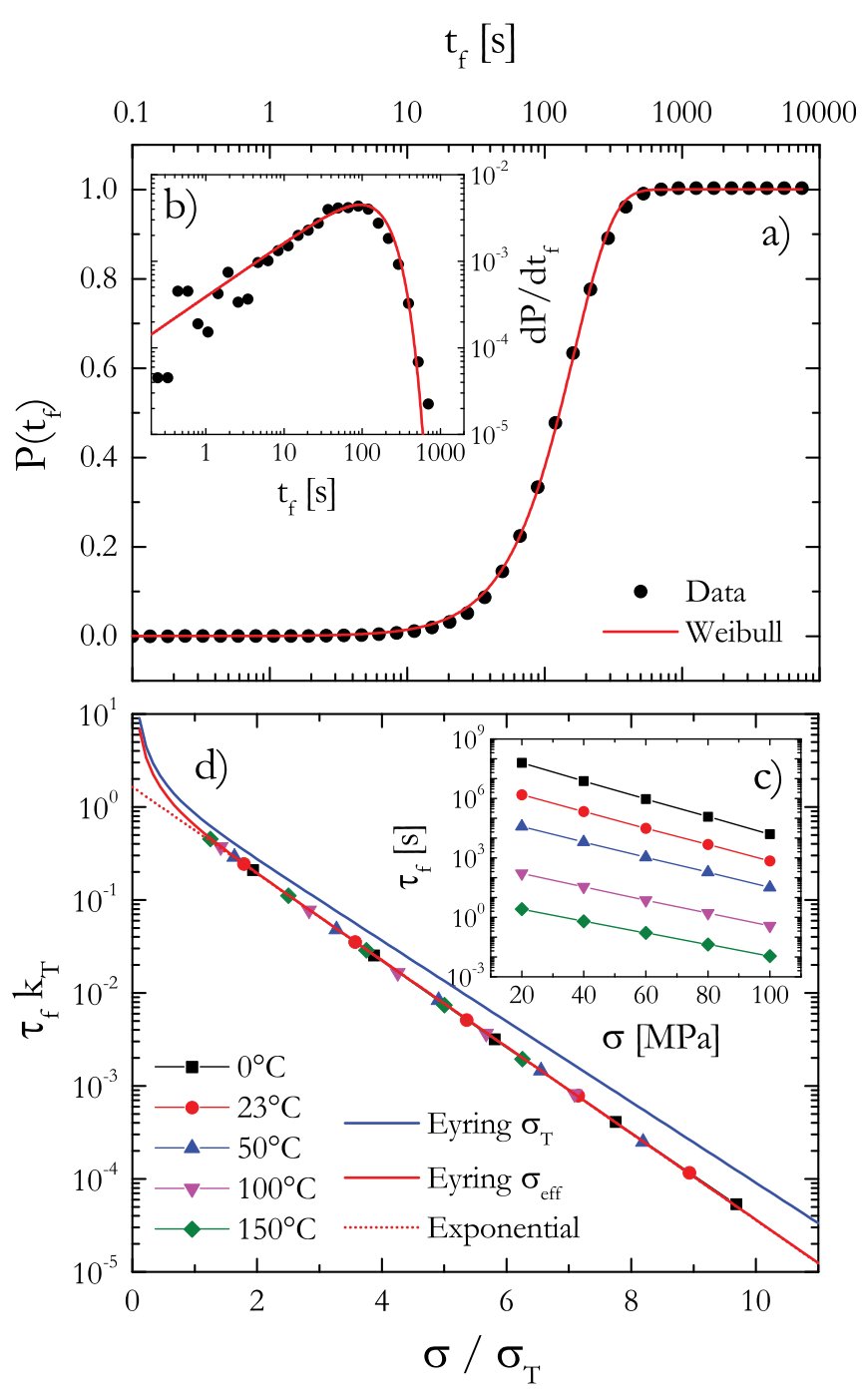

FIG. 9. (a) Symbols: cumulative failure time probability obtained by running 1000 simulations on equivalent samples under equal conditions ( $T=100^{\circ} \mathrm{C}, \sigma=20 \mathrm{MPa}$ ). Solid line: fit with Weibull distribution [Eq. (5)]. (b) Probability distribution of failure times. (c) Average failure time $\tau_{f}$ as a function of the applied stress. Different series represent different temperatures, from top to bottom: $0,23,50,100$, and $150{ }^{\circ} \mathrm{C}$. (d) Symbols: rescaled average failure time as a function of adimensional stress. Blue line: eyring model for homogeneous deformation. Red solid line: eyring model with stress concentration. Red dotted line: exponential fit.

described in terms of an effective reference stress $\sigma_{\text {eff }}$, slightly lower than $\sigma_{T}$.

By looking at creep failure time distributions, we can thus identify two main parameters characterizing sample connectivity. The first is a static property, just related to structural parameters, which can be evaluated in the homogeneous deformation (i.e., small $\tilde{\sigma}$ ) limit: in this regime, a sample which is better connected in this sense will withstand a larger number of bond breaking events, thus it will last longer under creep. We can quantify this property by looking at the intercept $\tilde{\tau}_{0}=k_{T} \tau_{0}$ of an exponential fit of the $\tilde{\tau}_{f}(\tilde{\sigma})$ plot [39]. However, there is also a second parameter, related to how efficiently the local stress is redistributed, which is a dynamic property. Following what was mentioned previously, we quantify this efficiency using the $\sigma_{\text {eff }} / \sigma_{T}$ ratio: $\tilde{\sigma}_{\text {eff }}=\sigma_{\text {eff }} / \sigma_{T}=1$ corresponds to the ideal case of homogeneous deformation, whereas a sample with $\tilde{\sigma}_{\text {eff }}<1$ will tend to localize the deformation, specially under large stresses.

Both $\tilde{\tau}_{0}$ and $\tilde{\sigma}_{\text {eff }}$ depend on sample morphology: as an example, in Fig. 10, we report those parameters for samples where the only varying parameter is the chain length $N$. In agreement with expectations, longer chains produce better connected samples: $\tilde{\tau}_{0}$ grows logarithmically with $N$, whereas $\tilde{\sigma}_{\text {eff }}$ increases only by a few percent in the small $N$ regime and then seems to saturate on a $N$-independent plateau value, possibly linked to the one observed experimentally (Fig. 3).

\section{Percolation threshold}

The interplay between morphology and connectivity can also be addressed in numerical simulations by studying the microscopic distribution of plastic events. For example, a direct link with the analytical model can be provided by the local fraction of associated physical bonds: this quantity suffers larger fluctuations for increasingly heterogeneous deformations, whereas its average value at failure represents the percolation threshold $A_{t h}$ introduced in Sec. IV.

As already discussed, $A_{t h}$ will depend both on sample morphology (samples that are initially better connected will be harder to disconnect) and on the localization of microscopic plasticity (if bond breaking activity is localized in one specific region it will be much more effective [32]): thus, as before, we expect that $A_{t h}$ shows both a morphology and a stress/temperature dependence. This is indeed the case, as it is represented in Fig. 11(a): lower values of $\tilde{\sigma}$ are associated to more homogeneous deformations, and thus to a lower threshold, and in an analogous way for a given $\tilde{\sigma}$ better connected samples (larger $N$ ) redistribute more efficiently the stress; thus, they also have lower $A_{t h}$ values.

Such a rich phenomenology is represented in a simpler way in Fig. 11(b), where a manual rescaling parameter $\sigma_{D}$ was introduced to collapse the percolation thresholds on a master-curve. The fact, far from obvious, that such a collapse can be obtained confirms that the important parameter

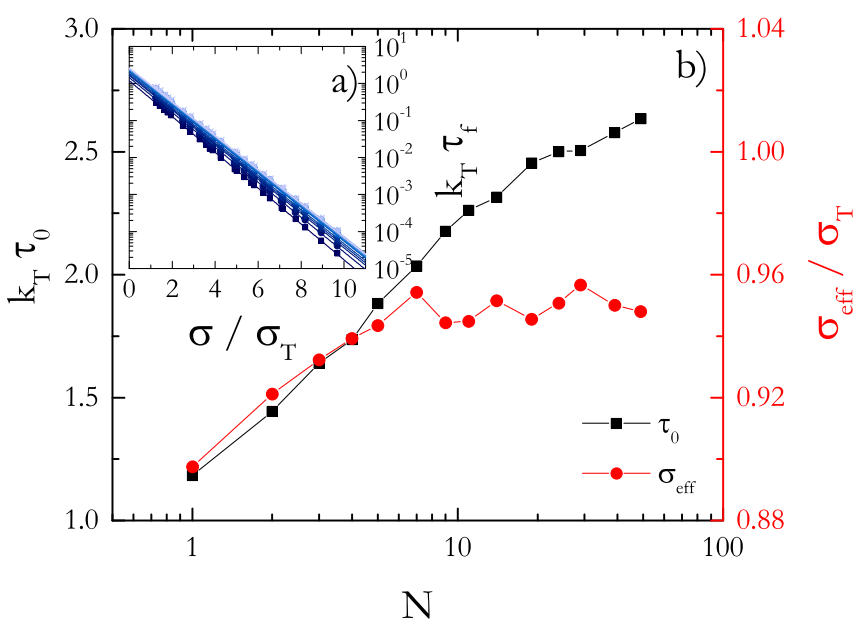

FIG. 10. (a) Symbols: a dimensional average failure time versus adimensional stress for various chain lengths, from dark to light: $N=1,2,3,4,5,7$, $9,12,15,20,25,30,40$, and $50 \mathrm{SB}$ segments. Lines: exponential fits, yielding an intercept $\left(\tilde{\tau}_{0}\right)$ and a slope $\tilde{\sigma}_{\text {eff }}$. (b) Fit parameters as a function of chain length. Black, left axis: intercept $\left(\tilde{\tau}_{0}\right)$; red, right axis: slope $\left(\tilde{\sigma}_{\text {eff }}\right)$. 

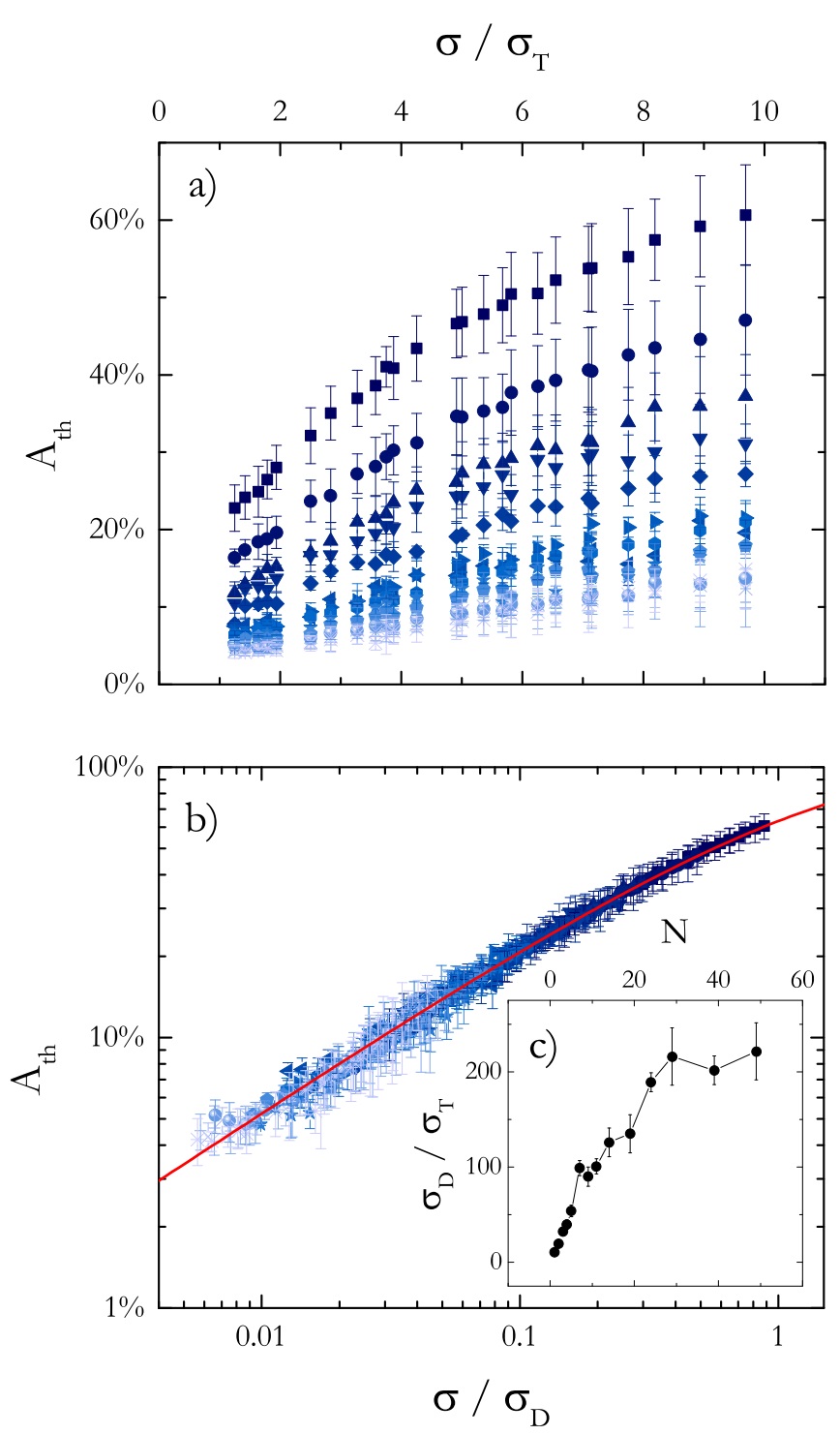

FIG. 11. (a) Fraction of residual physical bonds at failure, as a function of reduced stress for various chain lengths, increasing from dark to light (see Fig. 10 for the values). (b) Same data as panel (a), plotted against a rescaled stress variable. Red line: stretched exponential with stretching exponent $\alpha=0.63$ (c) Rescaling parameter $\sigma_{D}$ plotted as a function of chain length.

controlling the connectivity of a material is indeed how efficiently the local stress is concentrated around defects (here represented, for example, by chain ends that have lower coordination): the effect of macroscopic stress, temperature, and chain morphology can be interpreted in terms of their impact on stress concentration. This is quantified by the stress parameter $\sigma_{D}$, which is represented in Fig. 11(c) as a function of the chain length $N$. $\sigma_{D}$ increasing with $N$ tells us that, at a fixed temperature, a larger external stress is needed to produce the same stress heterogeneities in samples made of longer chains, or that, conversely, such samples deform more homogeneously (thus with a lower $A_{t h}$ threshold) under a given applied stress. Furthermore, Fig. 11(c) shows that the impact of chain length on stress concentration becomes negligible for the larger $N$ values, and this offers a practical tool to tackle the $M_{w}$ dependence of the failure strain observed in Fig. 3. In analogy with simulation results, we can interpret the first growth of $\varepsilon_{f}\left(M_{\omega}\right)$ as the sign that longer chains produce better connected samples, which have higher resistance under tension. In turn, the plateau of $\varepsilon_{f}$ observed in the large $M_{w}$ limit reminds the fact that connectivity is no longer improved significantly when chain length is increased beyond a given limit.

\section{Tensile test simulations}

We now turn in this final section to simulating strain rate imposed experiments, which represent a less natural framework for our model, but which can be more directly compared with the experimental data shown in Sec. III. Since our kinetic model does not contain any information about strain, we use stress as a function of time fitted to the experimental tensile curves as an input for numerical simulations.

Starting again from Fig. 2(a), we use the observation that stress seems to be roughly independent on strain rate to extract from experimental data a $\dot{\varepsilon}$-independent set of stressstrain curves $\sigma(\varepsilon, T)$. These will represent the starting point for our strain rate imposed simulations: for a given strain rate $\dot{\varepsilon}$, a time dependent stress $\sigma(t)=\sigma(\varepsilon=\dot{\varepsilon} t, T)$ is applied to the sample, in order to reproduce the one macroscopically experienced in the experiment. Whenever it was needed, a polynomial fit of the measured $\sigma(\varepsilon, T)$ curves allowed one to extrapolate the mechanical stresses beyond the experimental limit imposed by $\varepsilon_{f}$. In Fig. 2(b), the maximum elongations measured experimentally are compared to the one extracted by numerical simulations performed on similar conditions. The fair agreement obtained at different temperatures and strain rates can be considered as another indication that despite its intrinsic limitations the model is able to grasp the fundamental features of the sample failure mechanism.

Figure 12 shows, more in detail, the failure strain predicted by numerical simulations using as a starting point the experimental $\sigma(\varepsilon, T)$ curves of Fig. 1 (with the only exception of room temperature, because of strain hardening). In

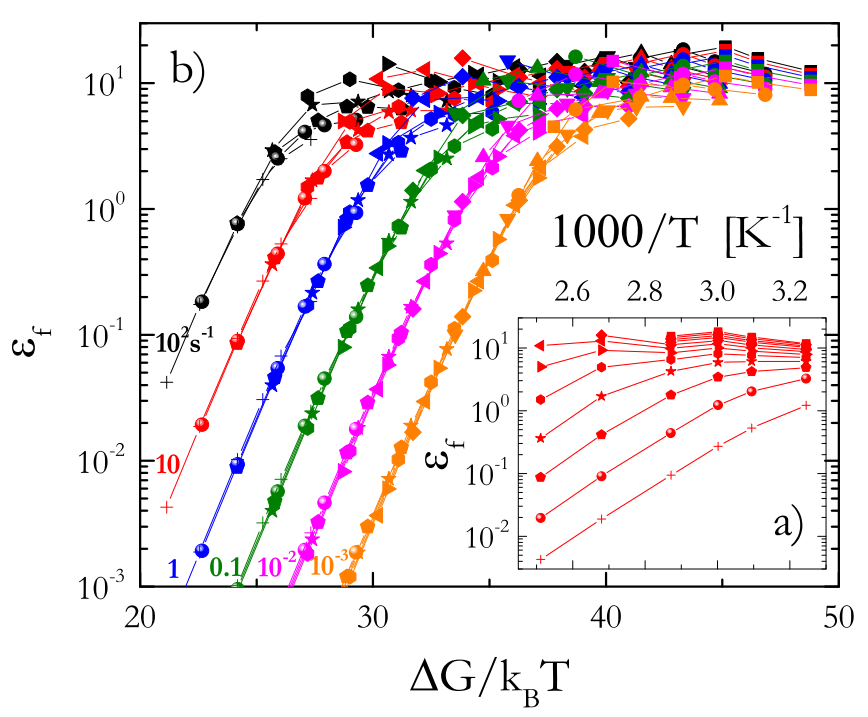

FIG. 12. (a) Elongation at break extracted from numerical simulations for different values of the activation energy $\Delta G$, from 80 to $120 \mathrm{~kJ} / \mathrm{mol}$, for a strain rate $\dot{\varepsilon}=10 \mathrm{~s}^{-1}$, plotted as a function of the inverse temperature. (b) Elongation at break obtained for different values of $\Delta G$ and $\dot{\varepsilon}$ (specified in the labels) plotted as a function of the rescaled variable $\Delta G / k_{B} T$. 
agreement with the analytical prediction of Eq. (4), we can again identify two distinct regimes: in the thermal regime, $\varepsilon_{f}$ grows linearly with $\dot{\varepsilon}$ and exponentially with $T^{-1}$, whereas in the stress-dominated regime, $\varepsilon_{f}$ reaches a plateau value that depends only weakly on $T$ and $\dot{\varepsilon}$.

Strain rate imposed simulations also represent a convenient framework to study the impact of model parameters $\left(\Delta G, v^{*}\right)$ on the observed failure strain. For example, we can take the same experimental tensile curves of Fig. 1 and use them as an input for a series of numerical simulations where only the activation energy $\Delta G$ is changed, spanning a narrow interval around the value extracted from the fitting procedure discussed in Sec. IV C. Figure 12(a) shows the resulting failure strain as a function of inverse temperature, for $\Delta G$ ranging from 80 to $120 \mathrm{~kJ} / \mathrm{mol}$ : we observe that $\varepsilon_{f}$ depends strongly on $\Delta G$, and this dependence is stronger at high temperatures. Figure 12(b) quantitatively addresses such dependence: the observed failure strains collapse on $\dot{\varepsilon}$-dependent mastercurves if plotted against the rescaled variable $\Delta G / k_{B} T$. This fact, confirmed by preliminary experimental results (not shown), also suggests that the activation energy $\Delta G$ is the most relevant parameter controlling the drop in extensibility at high temperatures. Besides its interest from a fundamental point of view, this may indicate the path toward the development of better performing TPEs, with a larger extensibility: indeed, for a fixed strain rate, it may be possible to tune $\Delta G$ (for example by modifying the chemistry of the HBs or by improving the HB crystal quality), until the drop in $\varepsilon_{f}$ falls outside the working temperature range.

\section{CONCLUSION}

In this paper we have introduced a kinetic model based on Erying rate equation to describe the breaking apart of physical bonds in a TPE. The sample is regarded as a transient network, whose failure is associated to the loss of connectivity. We apply the model in an analytical and numerically integrated form as well as in numerical simulations, to investigate how it captures the experimental results and to understand its implications for stress localization, with a particular focus on the role of sample morphology. This model captures the experimentally observed (1) drop in elongation at break with increasing temperature, (2) drop in elongation at break with decreasing strain rate (and relatively weaker dependence on strain rate relative to temperature), (3) and the increase in elongation at break with increasing molecular weight.

At the same time, the model neglects some aspects, namely all morphological modifications before fibrillar structure is formed, and the effect of interactions between SBs, either due to entanglements or to crystallization. This restricts the model validity to nonentangled systems in the large strain regime $(\varepsilon>100 \%)$ and for temperatures well above the glass transition temperature $T_{g}$ and below the melting temperature $T_{m}$. Another aspect that has been only marginally taken into account in the model is the relevance of the TPE softness. A more detailed investigation on samples with different $\mathrm{HB} / \mathrm{SB}$ ratios can offer a more complete perspective on the model validity and on its limitations.

From an experimental point of view, a direct observation of plasticity developed during deformation, for example, with infrared spectroscopy [17], infrared dichroism [5,69-71], light scattering [72], x-ray scattering $[15,17,18,39,73-76]$, NMR [77-80], or other optical techniques [81-84], would provide valuable information about the percolation threshold parameter that our model points out as a practical way to characterize sample connectivity. In particular, space resolved techniques [72,81-83,85] might represent powerful tools to investigate the spatial localization of bond-breaking events, which strongly influences the mechanical properties [86], as it is shown in our simulations. Experimentally accessing this quantity for samples with different microscopic architectures could open new perspectives to the development of more advanced materials.

Finally, our work shows that elongation at break is strongly dependent on the kinetics of physical bonds, which determine the onset of the thermal regime, where extensibility is strongly reduced with increasing temperature. Therefore, future development should consider kinetics as the key parameter to tune in order to obtain well-performing TPEs in the whole working temperature range.

\section{ACKNOWLEDGMENTS}

The work leading to these results has received funding from the People Programme (Marie Sklodowska-Curie Actions) of the European Union's Seventh Framework Programme (FP7/2007-2013) under REA Grant Agreement No. 607937 SUPOLEN project. The authors also thankfully acknowledge Wilco Appel for the synthesis of the samples studied, Patrick van Soelen for help with instrumentation, Sophia Ganzeboom for mechanical testing, and Carel Fitié for stimulating discussions.

\section{APPENDIX A: BOX SIZE EFFECTS IN NUMERICAL SIMULATIONS}

Numerical simulations shown in the text are performed on cubic samples of volume $V=100^{3}$ sites. This size is chosen in order to minimize finite size effects while keeping a reasonable simulation time. Finite simulation volume effects were addressed by changing both sample volume and aspect ratio (i.e., by changing, at fixed volume, the sample size along the strain direction).

As a first parameter potentially affected by finite box size we studied the single chain conformation, by looking at the average chain end-to-end distance $\left\langle R_{e e}\right\rangle$. Figure 13 shows this quantity for different chain lengths and different (cubic) box sizes. The observed value follows the one expected for a polymer in theta solvent [87], until a deviation is observed as soon as $\left\langle R_{e e}\right\rangle$ exceeds half the lateral box size. In our simulations, we chose to work with chains short enough to be well below this regime.

More importantly than chain conformation, we addressed the sample volume dependence of creep failure time. This is a well-known problem, experimentally investigated by 


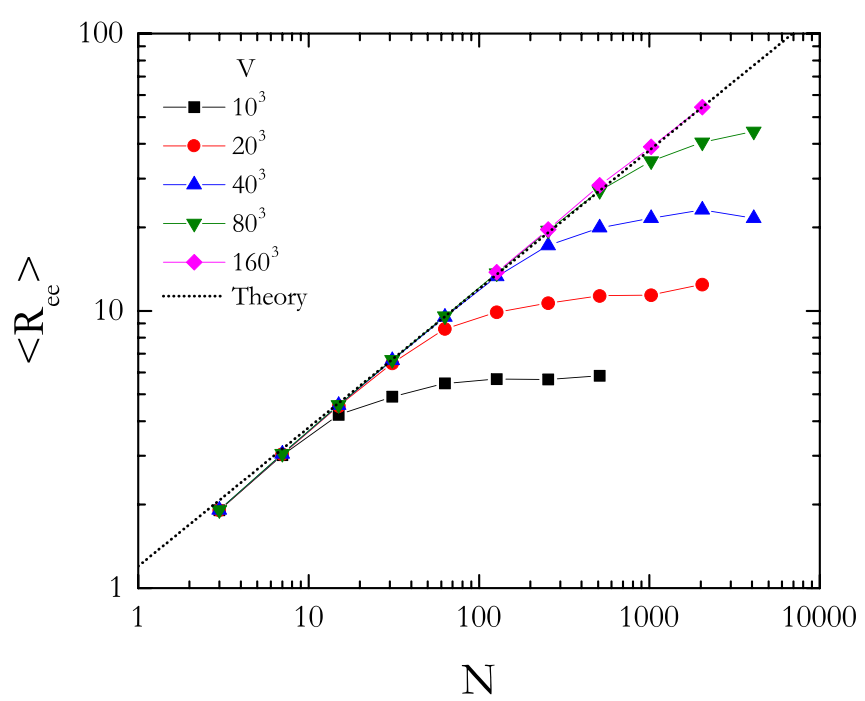

FIG. 13. Chain end to end distance (in units of the lattice size) as a function of the number $N$ of SBs. Different series refer to simulations performed in cubic boxes of different sizes: the number of sites along one edge of the cubic box grows from 10 to 160 as specified in the legend.

looking at the tensile strength of wires since XV century, when Leonardo da Vinci first observed that longer wires were weaker that shorter ones $[88,89]$. Indeed, in Weibull theory [63], the failure probability is assumed to grow exponentially with the sample volume (the general argument being that larger samples contain a larger amount of critical defects [66]). This is in good agreement with numerical simulations, as it is shown in Fig. 14: under the same experimental conditions, larger samples fail earlier. More precisely, for a fixed transversal section (different colors in Fig. 14), elongated samples are more fragile, and the failure time decreases as $V^{-1 / \beta}$ [63]. This happens because thinner samples are more heterogeneous (lower $\beta$ ), thus more prone to

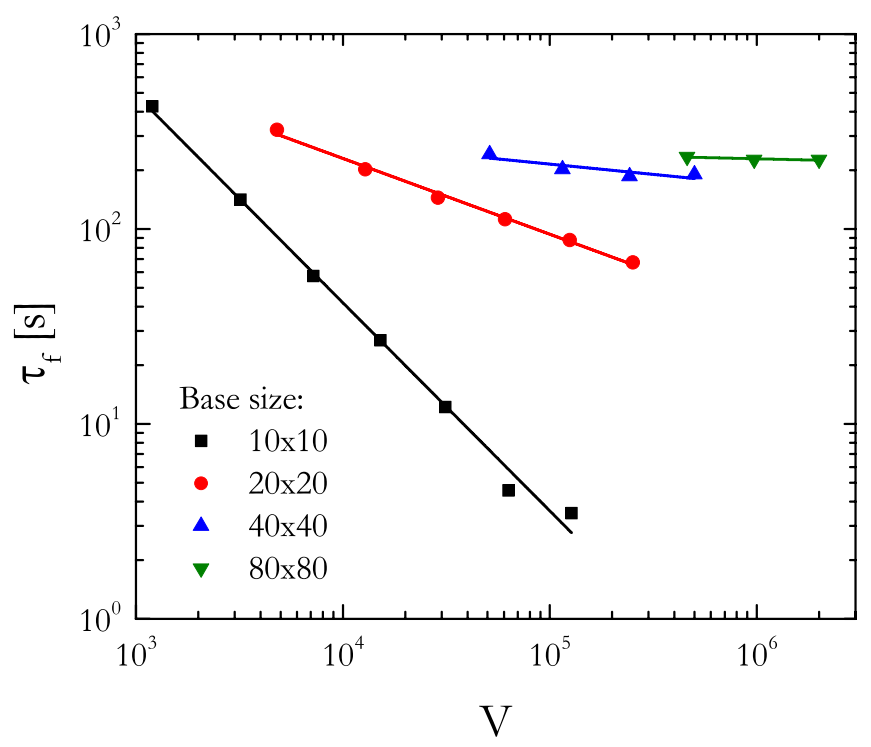

FIG. 14. Symbols: average creep failure time measured as a function of sample size ( $V$ represents the volume of the simulation box, in units of the cubic lattice size), under equivalent conditions (temperature $T=60^{\circ} \mathrm{C}$, stress $\sigma / \sigma_{T}=12$ ) for a system of chains with $N=3$ SBs per chain. Lines: power law fits with slope fixed to the expected $-1 / \beta$ value, with $\beta=0.9$, $2.6,9.5$, and 40 (extracted by a Weibull fit of failure time distributions at different sample sizes). stress concentration [90], which reflects in a sharper dependence of failure time on sample volume. By contrast, for sample thicknesses large enough, like the one used in this paper, the probability of finding a critical defect just because of random chain disposition is significantly suppressed, and the failure dynamics will be roughly independent on sample size.

\section{APPENDIX B: CREEP COMPLIANCE}

Numerical simulations presented in Sec. V do not contain any information on macroscopic strain: the only relevant variables are stress and time. That is why creep compliance cannot be accessed, and we must limit ourselves to analyze the failure time distribution. In order to overcome this limitation, a stressstrain relation is needed, at least for one single SB chain. For example, one can start assuming that each SB acts as an entropic spring, with a given Young modulus $E$, and use this assumption to link the local stress $\sigma_{l o c}(k, t)$ experienced by plane $k$ to a local strain $\varepsilon_{l o c}(k, t)$. All SBs being identical, the local stress and strains will be different from the macroscopic ones just because the number $n_{S B}(k, t)$ of SBs bridging any $k$ plane with the nearest ones is not constant. Indeed, $n_{S B}(k, t)$ is initially fluctuating, and it evolves toward even more heterogeneous configurations because of stress concentration.

Because of linearity the total strain will be the arithmetic average of local strains, whereas the macroscopic true stress will be by definition related to local stresses via a harmonic average:

$\sigma_{\text {True }}(t)=\left[\left\langle\frac{1}{\sigma_{l o c}(k, t)}\right\rangle_{k}\right]^{-1} \leq\left\langle\sigma_{l o c}(k, t)\right\rangle_{k}=E\left\langle\varepsilon_{l o c}(k, t)\right\rangle_{k}=E \varepsilon$.

The inequality is a well-known theorem in mathematics [91]: in particular, since the ratio between arithmetic and harmonic average increases if the local stress profile gets increasingly heterogeneous [92], creep compliance under a constant external stress increases when connectivity is progressively lost, until failure occurs. For the same reason, according to this model we should expect creep compliance at break to show only weak dependence on stress and temperature, which is in good agreement with experimental data [cf. Fig. 8(a) for the stress dependence] [64].

Also in this case, maximum extensibility depends on sample morphology (for example, chain molar mass). However, it is worth to mention that, once plotted against the rescaled stress variable $\sigma / \sigma_{D}$, again simulation results for $\varepsilon_{f}$ in creep tend to collapse on a master-curve: this is again because creep extension is strictly linked to the heterogeneity of the deformation.

\section{References}

[1] Callister, W. D., and D. G. Rethwisch, Materials Science and Engineering: An Introduction, 9th ed. (Wiley, Hoboken, NJ, 2014).

[2] Handbook of Condensation Thermoplastic Elastomers, edited by S. Fakirov (Wiley-VCH Verlag GmbH and Co, Weinheim, 2005).

[3] Shackelford, J. F., Introduction to Materials Science for Engineers, 8th ed. (Pearson, Boston, 2015). 
[4] Spychaj, T., M. Kacperski, and A. Kozlowska, "Condensation and addition thermoplastic elastomers: Recycling aspects," in Handbook of Condensation Thermoplastic Elastomers (Wiley-VCH Verlag $\mathrm{GmbH}$ and Co, Weinheim, 2005), Chap. 19, pp. 567-592.

[5] Gaymans, R. J., "Segmented copolymers with monodisperse crystallizable hard segments: Novel semi-crystalline materials," Prog. Polym. Sci. 36, 713-748 (2011).

[6] Fakirov, S., and T. Gogeva, "Poly(ether/ester)s based on poly(butylene terephthalate) and poly(ethylene glycol), 1. Poly (ether/ester)s with various polyether: Polyester ratios," Makromol. Chem. 191, 603-614 (1990).

[7] Fakirov, S., and T. Gogeva, "Poly(ether/ester)s based on poly(butylene terephthalate) and poly(ethylene glycol), 2. Effect of polyether segment length," Macromol. Chem. Phys. 191, 615-624 (1990).

[8] Fakirov, S., A. A. Apostolov, P. Boeseke, and H. G. Zachmann, "Structure of segmented poly(ether ester)s as revealed by synchrotron radiation," J. Macromol. Sci., Part B 29, 379-395 (1990).

[9] Stribeck, N., A. A. Apostolov, H. G. Zachmann, C. Fakirov, M. Stamm, and S. Fakirov, "Small angle x-ray scattering of segmented block copolyetheresters during stretching," Int. J. Polym. Mater. 25, 185-200 (1994).

[10] Deschamps, A. A., D. W. Grijpma, and J. Feijen, "Poly(ethylene oxide)/poly(butylene terephthalate) segmented block copolymers: The effect of copolymer com-position on physical properties and degradation behavior," Polymer 42, 9335-9345 (2001).

[11] Baeza, G. P., A. Sharma, A. Louhichi, L. Imperiali, W. P. J. Appel, C. F. C. Fitié, M. P. Lettinga, E. Van Ruymbeke, and D. Vlassopoulos, "Multiscale organization of thermoplastic elastomers with varying content of hard segments," Polymer 107, 89-101 (2016).

[12] Sijbrandi, N. J., A. J. Kimenai, E. P. C. Mes, R. Broos, G. Bar, M. Rosenthal, Y. Odarchenko, D. A. Ivanov, P. J. Dijkstra, and J. Feijen, "Synthesis, morphology, and properties of segmented poly(ether amide)s with uniform oxalamide-based hard segments," Macromolecules 45, 3948-3961 (2012).

[13] Fakirov, S., "Poly(ether ester) thermoplastic elastomers: Phase and deformation behavior on the nano- and microlevel," in Handbook of Condensation Thermoplastic Elastomers (Wiley-VCH Verlag $\mathrm{GmbH}$ and Co, Weinheim, 2005), Chap. 5, pp. 167-196.

[14] Apostolov, A. A., and S. Fakirov, "Effect of the block length on the deformation behavior of polyetheresters as revealed by small-angle x-ray scattering," J. Macromol. Sci., Part B 31, 329-355 (1992).

[15] Stribeck, N., D. Sapoundjieva, Z. Denchev, A. A. Apostolov, H. G. Zachmann, M. Stamm, and S. Fakirov, "Deformation behavior of pol$\mathrm{y}$ (ether ester) copolymer as revealed by small- and wide-angle scattering of x-ray radiation from synchrotron," Macromolecules 30, 1329-1339 (1997).

[16] Wu, L., T. P. Lodge, and F. S. Bates, "Effect of block number on multiblock copolymer lamellae alignment under oscillatory shear," J. Rheol. 49, 1231-1252 (2005).

[17] Yeh, F., B. S. Hsiao, B. B. Sauer, S. Michel, and H. W. Siesler, "Insitu studies of structure development during deformation of a segmented poly(urethaneurea) elastomer," Macromolecules 36, 1940-1954 (2003).

[18] Toki, S., B. S. Hsiao, S. Kohjiya, M. Tosaka, A. H. Tsou, and S. Datta, "Synchrotron X-ray studies of vulcanized rubbers and thermoplastic elastomers," Rubber Chem. Technol. 79, 460-488 (2006).

[19] Niesten, M. C. E. J., and R. J. Gaymans, "Tensile and elastic properties of segmented copolyesteramides with uniform aramid units," Polymer 42, 6199-6207 (2001).

[20] Biemond, G. J. E., J. Feijen, and R. J. Gaymans, "Tensile properties of segmented block copolymers with monodisperse hard segments," J. Mater. Sci. 43, 3689-3696 (2008).
[21] Ward, I. M., and J. Sweeney, An Introduction to the Mechanical Properties of Solid Polymers, 2nd ed. (Wiley, Chichester, 2004).

[22] The Physics of Glassy Polymers edited by R. N. Haward and R. J. Young (Springer, Netherlands, 1997).

[23] Smith, T. L., "Tensile strength of polyurethane and other elastomeric block copolymers," Rubber Chem. Technol. 49, 64-84 (1976).

[24] Smith, T. L., "Strength of elastomers-A perspective," Polym. Eng. Sci. 17, 129-143 (1977).

[25] Yamakawa, H., and H. Miyata, "High performance thermoplastic aramid elastomers: Synthesis, properties, and applications," in Handbook of Condensation Thermoplastic Elastomers (Wiley-VCH Verlag GmbH and Co, Weinheim, 2005), Chap. 5, pp. 141-166.

[26] Gabriëlse, W., M. Soliman, and K. Dijkstra, "Microstructure and phase behavior of block copoly(ether ester) thermoplastic elastomers," Macromolecules 34, 1685-1693 (2001).

[27] Marechal, E., "Polycondensation reactions in thermoplastic elastomer chemistry: state of the art, trends, and future developments," in Handbook of Condensation Thermoplastic Elastomers (Wiley-VCH Verlag GmbH and Co, Weinheim, 2005), Chap. 2, pp. 33-66.

[28] Stribeck, N., S. Fakirov, A. A. Apostolov, Z. Denchev, and R. Gehrke, "Deformation behavior of PET, PBT and PBT-based thermoplastic elastomers as revealed by SAXS from synchrotron," Macromol. Chem. Phys. 204, 1000-1013 (2003).

[29] Niesten, M. C. E. J., S. Harkema, E. Van der Heide, and R. J. Gaymans, "Structural changes of segmented copolyetheresteramides with uniform aramid units induced by melting and deformation," Polymer 42, 1131-1142 (2001).

[30] Versteegen, R. M., R. Kleppinger, R. P. Sijbesma, and E. W. Meijer, "Properties and morphology of segmented copoly(ether urea)s with uniform hard segments," Macromolecules 39, 772-783 (2006).

[31] Deplace, F., Z. Wang, N. A. Lynd, A. Hotta, J. M. Rose, P. D. Hustad, J. Tian, H. Ohtaki, G. W. Coates, F. Shimizu, K. Hirokane, F. Yamada, Y.-W. Shin, L. Rong, J. Zhu, S. Toki, B. S. Hsiao, G. H. Fredrickson, and E. J. Kramer, "Processing-structure-mechanical property relationships of semicrystalline polyolefin-based block copolymers," J. Polym. Sci., Part B 48, 1428-1437 (2010).

[32] Tanaka, F., and S. F. Edwards, "Viscoelastic properties of physically crosslinked networks. 1. Transient network theory," Macromolecules 25, 1516-1523 (1992).

[33] Mora, S., "The kinetic approach to fracture in transient networks," Soft Matter 7, 4908-4917 (2011).

[34] Ligoure, C., and S. Mora, "Fractures in complex fluids: The case of transient networks," Rheol. Acta 52, 91-114 (2013).

[35] Bauwens-Crowet, C., J. C. Bauwens, and G. Homes, "Tensile yieldstress behavior of glassy polymers," J. Polym. Sci., Part A 7, 735-742 (1969).

[36] Hasan, O. A., M. C. Boyce, X. S. Li, and S. Berko, "An investigation of the yield and postyield behavior and corresponding structure of poly(methyl methacrylate), J J. Polym. Sci., Part B 31, 185-197 (1993).

[37] Buckley, C. P., and D. C. Jones, "Glass-rubber constitutive model for amorphous polymers near the glass transition," Polymer 36, 3301-3312 (1995).

[38] Buckley, C., "Deformation of thermosetting resins at impact rates of strain. Part 2: Constitutive model with rejuvenation,” J. Mech. Phys. Solids 52, 2355-2377 (2004).

[39] van Erp, T. B., D. Cavallo, G. W. M. Peters, and L. E. Govaert, "Rate-, temperature-, and structure-dependent yield kinetics of isotactic polypropylene," J. Polym. Sci., Part B 50, 1438-1451 (2012).

[40] Roetling, J. A., "Yield stress behaviour of isotactic polypropylene," Polymer 7, 303-306 (1966). 
[41] Roetling, J. A., "Yield stress behaviour of polymethylmethacrylate," Polymer 6, 311-317 (1964).

[42] van Erp, T. B., C. T. Reynolds, T. Peijs, J. A. W. van Dommelen, and L. E. Govaert, "Prediction of yield and long-term failure of oriented polypropylene: Kinetics and anisotropy,” J. Polym. Sci., Part B 47, 2026-2035 (2009).

[43] Coleman, B. D., "Application of the theory of absolute reaction rates to the creep failure of polymeric filaments," J. Polym. Sci. 20, 447-455 (1956).

[44] Bueche, F., "Tensile strength of plastics below the glass temperature," J. Appl. Phys. 28, 784-787 (1957).

[45] Bueche, F., "Tensile strength of plastics: Effects of flaws and chain relaxation,” J. Appl. Phys. 29, 1231-1234 (1958).

[46] Janssen, R. P. M., L. E. Govaert, and H. E. H. Meijer, "An analytical method to predict fatigue life of thermoplastics in uniaxial loading: Sensitivity to wave type, frequency, and stress amplitude," Macromolecules 41, 2531-2540 (2008).

[47] Visser, H. A., T. C. Bor, M. Wolters, T. A. P. Engels, and L. E. Govaert, "Lifetime assessment of load-bearing polymer glasses: An analytical framework for ductile failure," Macromol. Mater. Eng. 295, 637-651 (2010).

[48] Drozdov, A. D., and J. deC. Christiansen, "Creep failure of polypropylene: Experiments and constitutive modeling," Int. J. Fract. 159, 63-79 (2009).

[49] Matz, D. J., W. G. Guldemond, and S. L. Cooper, "Delayed yielding in glassy polymers,” J. Polym. Sci., Part B 10, 1917-1930 (1972).

[50] Eyring, H., "Viscosity, plasticity and diffusion as examples of absolute reaction rates," J. Chem. Phys. 4, 283-291 (1936).

[51] Green, M. S., and A. V. Tobolsky, "A new approach to the theory of relaxing polymeric media,” J. Chem. Phys. 14, 80-92 (1946).

[52] Ree, T., and H. Eyring, "Theory of non-Newtonian flow. I. Solid plastic system,” J. Appl. Phys. 26, 793-800 (1955).

[53] Ree, T., and H. Eyring, "Theory of non-Newtonian flow. II. Solution system of high polymers," J. Appl. Phys. 26, 800-809 (1955).

[54] Tervoort, T. A., E. T. J. Klompen, and L. E. Govaert, "A multimode approach to finite, threedimensional, nonlinear viscoelastic behavior of polymer glasses," J. Rheol. 40, 779-797 (1996).

[55] Klompen, E. T. J., T. A. P. Engels, L. C. A. van Breemen, P. J. G. Schreurs, L. E. Govaert, and H. E. H. Meijer, "Quantitative prediction of long-term failure of polycarbonate," Macromolecules 38, 7009-7017 (2005).

[56] Klompen, E. T. J., T. A. P. Engels, L. E. Govaert, and H. E. H. Meijer, "Modeling of the postyield response of glassy polymers: Influence of thermomechanical history," Macromolecules 38, 6997-7008 (2005).

[57] Zhurkov, S. N., "Kinetic concept of the strength of solids," Int. J. Fract. Mech. 1, 311-323 (1965).

[58] Zhurkov, S. N., and S. A. Abasov, "The temperature and the time dependence of the strength of polymer yarns," Polym. Sci., Ser. A 41, 1276-1282 (1999).

[59] Chen, K., and K. S. Schweizer, "Stress-enhanced mobility and dynamic yielding in polymer glasses," Europhys. Lett. 79, 26006 (2007).

[60] Wu, J. J., and C. P. Buckley, "Plastic deformation of glassy polystyrene: A unified model of yield and the role of chain length," J. Polym. Sci., Part B 42, 2027-2040 (2004).

[61] Termonia, Y., "Molecular model for the mechanical properties of elastomers. 1. Network formation and role of entanglements," Macromolecules 22, 3633-3638 (1989).

[62] Narisawa, I., M. Ishikawa, and H. Ogawa, "Delayed yielding of polycarbonate under constant load," J. Polym. Sci. 16, 1459-1470 (1978).

[63] Weibull, W., "A statistical theory of the strength of materials," Ingeniors Vetenskaps Akademiens Handlingar 151, 1939.
[64] Vas, L. M., and P. Bakonyi, "Estimating the creep strain to failure of PP at different load levels based on short term tests and Weibull characterization,” Express Polym. Lett. 6, 987-996 (2012).

[65] Nakada, M., and Y. Miyano, "Statistical creep failure time of unidirectional CFRP," Exp. Mech. 56, 653-658 (2016).

[66] Coleman, B. D., "Statistics and time dependence of mechanical breakdown in fibers," J. Appl. Phys. 29, 968-983 (1958).

[67] Liu, A. J., and S. R. Nagel, "Nonlinear dynamics: Jamming is not just cool any more," Nature 396, 21-22 (1998).

[68] Haxton, T. K., and A. J. Liu, "Activated dynamics and effective temperature in a steady state sheared glass," Phys. Rev. Lett. 99, 195701 (2007).

[69] Seymour, R. W., G. M. Estes, and S. L. Cooper, "Infrared studies of segmented polyurethan elastomers. I. Hydrogen bonding," Macromolecules 3, 579-583 (1970).

[70] Seymour, R. W., A. E. Allegrezza, Jr., and S. L. Cooper, "Segmental orientation studies of block polymers. I. Hydrogen-bonded polyurethanes," Macromolecules 6, 896-902 (1973).

[71] Seymour, R. W., and S. L. Cooper, "Viscoelastic properties of polyurethane block polymers," Rubber Chem. Technol. 47, 19-31 (1974).

[72] Nagazi, M.-Y., G. Brambilla, G. Meunier, P. Marguers, J.-N. Périé, and L. Cipelletti, "Space-resolved diffusing wave spectroscopy measurements of the macroscopic deformation and the microscopic dynamics in tensile strain tests," Opt. Lasers Eng. 88, 5-12 (2017).

[73] Bauer, T., J. Oberdisse, and L. Ramos, "Collective rearrangement at the onset of flow of a polycrystalline hexagonal columnar phase," Phys. Rev. Lett. 97, 258303 (2006).

[74] Addiego, F., S. Patlazhan, K. Wang, S. André, S. Bernstorff, and D. Ruch, "Time-resolved small-angle x-ray scattering study of void fraction evolution in high-density polyethylene during stress unloading and strain recovery: SAXS study of void fraction evolution in HDPE," Polym. Int. 64, 1513-1521 (2015).

[75] Farge, L., S. André, F. Meneau, J. Dillet, and C. Cunat, "A common multiscale feature of the deformation mechanisms of a semicrystalline polymer," Macromolecules 46, 9659-9668 (2013).

[76] Farge, L., J. Boisse, J. Dillet, S. André, P.-A. Albouy, and F. Meneau, "Wide-angle x-ray scattering study of the lamellar/fibrillar transition for a semi-crystalline polymer deformed in tension in relation with the evolution of volume strain," J. Polym. Sci., Part B 53, 1470-1480 (2015).

[77] Loo, L. S., R. E. Cohen, and K. K. Gleason, "Chain mobility in the amorphous region of nylon 6 observed under active uniaxial deformation," Science 288, 116-119 (2000).

[78] Schaefer, D. J., R. J. Schadt, K. H. Gardner, V. Gabara, S. R. Allen, and A. D. English, "Microscopic dynamics and macroscopic mechanical deformation of poly(p-phenyleneterephthalamide) fibers," Macromolecules 28, 1152-1158 (1995).

[79] Hansen, M. T., C. Boeffel, and H. W. Spiess, "Phenylene motion in polycarbonate: Influence of tensile stress and chemical modification," Colloid Polym. Sci. 271, 446-453 (1993).

[80] Loo, L. S., R. E. Cohen, and K. K. Gleason, "Deuterium nuclear magnetic resonance of phenol-d5 in nylon 6 under active uniaxial deformation," Macromolecules 32, 4359-4364 (1999).

[81] Lee, H.-N., K. Paeng, S. F. Swallen, and M. D. Ediger, "Dye reorientation as a probe of stress-induced mobility in polymer glasses," J. Chem. Phys. 128, 134902 (2008).

[82] Lee, H.-N., K. Paeng, S. F. Swallen, and M. D. Ediger, "Direct measurement of molecular mobility in actively deformed polymer glasses," Science 323, 231-234 (2009). 
[83] André, S., C. Baravian, N. Renault, and C. Cunat, "In situ mechanical characterization of polymers with the association of three optical techniques," Appl. Phys. Lett. 91, 071919 (2007).

[84] Baravian, C., S. André, N. Renault, N. Moumini, and C. Cunat, "Optical techniques for in situ dynamical investigation of plastic damage," Rheol. Acta 47, 555-564 (2008).

[85] André, S., N. Renault, Y. Meshaka, and C. Cunat, "From the thermodynamics of constitutive laws to the thermomechanical experimental characterization of a semicrystalline polymer from IR imaging," Continuum Mech. Thermodyn. 24, 1-20 (2012).

[86] Ediger, M. D., "Spatially heterogeneous dynamics in supercooled liquids," Annu. Rev. Phys. Chem. 51, 99-128 (2000).

[87] Colby, R. H., and M. Rubinstein, Polymer Physics (Oxford University, Weinheim, 2003).
[88] Timoshenko, S. P., History of Strength of Materials (McGraw-Hill, Weinheim, 1953).

[89] Lund, J. R., and J. P. Byrne, "Leonardo Da Vinci's tensile strength tests: Implications for the discovery of engineering mechanics," Civil Eng. Syst. 18, 243-250 (2001).

[90] Amaniampong, G., and C. J. Burgoyne, "Monte-Carlo simulations of the time dependent failure of bundles of parallel fibres," Eur. J. Mech., A 2, 243-266 (1996).

[91] Xia, D.-F., S.-L. Xu, and F. Qi, "A proof of the arithmetic meangeometric mean-harmonic mean inequalities," Res. Rep. Collect. 2, 85-87 (1999).

[92] Limbrunner, J. F., R. M. Vogel, and L. C. Brown, "Estimation of harmonic mean of a lognormal variable," J. Hydrol. Eng. 5, 59-66 (2000). 